Jan M. Boyde, Patrick C. Le Clercq, Massimiliano Di Domenico, Manfred Aigner, Extension of the turbulent flame speed closure model to ignition in multiphase flows, Combust. Flame 160 (2012) 351-365

The original publication is available at www.elsevier.com

http://dx.doi.org/10.1016/j.combustflame.2012.10.006 


\title{
Extension of the Turbulent Flame Speed Closure Model to Ignition in Multiphase Flows.
}

\author{
Jan Michael Boyde ${ }^{1}$, Patrick C. Le Clercq ${ }^{1}$, Massimiliano Di Domenico ${ }^{1}$, Manfred Aigner ${ }^{1}$ \\ ${ }^{1}$ DLR Stuttgart (German Aerospace Center), Pfaffenwaldring 38-40, 70569 Stuttgart, Germany
}

\begin{abstract}
An extension of the Turbulent Flame Speed Closure model rendering the model applicable to multiphase flow and ignition is presented. As formerly no coupling between reaction progress variable and enthalpy was existent, except through the temperature dependency of the laminar flame speed, an adaptation is proposed which offers an interface to initiate the combustion process. The modification to incorporate multiphase conditions is achieved by substituting the mixture fraction variable as representation of the composition in the original implementation of the Turbulent Flame Speed Closure model with independent species. Source terms to correlate the species progress to the reaction progress variable are derived in this work. The additional transport equations serve a higher generality of the model and enable the proper treatment of vaporizing fuel droplets. It is demonstrated that limitations which arise in the standard formulation of the model, stemming from differences in the transport equation for the reaction progress variable and the mixture fraction, are addressed and resolved by the new approach. Regarding the initiation of the flame, an additional source term for the reaction progress variable is introduced, which relates the reaction progress to the auto-ignition time. This allows the development of the flame without imposing artificial boundary conditions. The correct model behavior is established by means of a series of widely used test cases. The results of these simulations show that the model's potential to predict flame growth and more generally the flame evolution as a function of time and space is preserved. At the same time more sophisticated test case boundary conditions involving multiphase conditions and variable inflows in terms of composition can be incorporated. As a thorough assessment of the extended model capabilities, a multiphase lab scale set-up, which provides a comprehensive data set, is presented. The good agreement of the obtained results underline the range of applicability of the extended model and its accuracy, albeit its simplicity, for multiphase conditions.
\end{abstract}

Keywords:

Turbulent Flame Speed Closure model, Multiphase Flow, Ignition, Flame Propagation

\section{Introduction}

Low-order methods for the treatment of combustion processes in numerical simulations have a long history. With low-order we refer to combustion models in contrast to those using a detailed description of the gas phase chemistry, e.g. Di Domenico et al. [1], which considers complex kinetic schemes and requires the transport of a multitude of species. Indeed, since the development of global reaction schemes, e.g. Westbrook and Dryer [2], the use of such models is widely acknowledged. Concurrently, the demand of numerical work for investigating problems involving complex fluid dynamics has increased. Due to the improvement made in LES modeling [3], [4] a large part of modeling strategies focuses on implementing a multiphase combustion model within a LES context to simulate full scale gas turbine combustion chambers, [5-7]. However, as this essentially proves very costly in terms of computation time, a large number of calculations on supercomputers is usually out of scope. Specifically, studies involving parameter variations are predestined for a fast and effi- cient computation for which simplified turbulence modeling (e.g. URANS) and simplified combustion models such as the eddy dissipation concept [8] or the flamelet model [9] can be utilized. However, there are certain aspects of combustor development, also demanding a large number of simulations, as for instance the optimum position of an igniter or more generally, estimating lean blow out [10], which pose special requirements on the combustion modeling capabilities. For resolving ignition processes, an accurate description of the flame propagation is necessary, see e.g. Levebvre [11] whereas the simulation of lean blow out requires a precise prediction of the flame quenching phenomenon [12]. Thus, there is still a great need for low-order combustion models to be able to deal with the modern challenges of aero-engine design.

Within the context of igniter location, a significant number of experimental investigations which focus on determining the ignition probability within simplified geometries have been recently published [13-17]. The starting point in such series of experiments is marked with the analysis of the basic gaseous jet-flame configuration [15] 
for the development of numerical tools. Then, technically relevant applications such as a swirl-stabilized spray flame [17] are investigated. The interest of aircraft's engine original equipment manufacturers (OEM) regarding this topic is demonstrated through studies published by Stow et al. [18], Mosbach et al. [19] and Fyffe et al. [10]. With the combustion systems' concept changing from rich toward lean primary zone combustion in order to address policy measures regarding pollutant emissions reduction, in particular nitrogen oxides $\left(\mathrm{NO}_{\mathrm{x}}\right)$, the issue of ignitability of the engine is more pronounced. Actually, lean-burn low $\mathrm{NO}_{\mathrm{x}}$ combustors have an operating envelope closer to the stability limits with respect to rich-burn systems. Specifically, high-altitude relight and cold start where adverse conditions prevail are thoroughly investigated experimentally in dedicated facilities during OEMs lean combustion system research programs [10]. Thus, there is a growing interest in dealing with this matter at an early stage of the combustor design cycle entraining a demand for numerical tools to support the development and allow a multitude of simultaneous computations. In this context, ad-hoc ignition models as proposed by Neophytou et al. [20] and Weckering et al. [21] were recently developed.

The numerical work which has been done with more complex models concerning the ignition probability of some of the test cases [13-17] has shown that a good agreement for distinct points can be achieved in terms of the ignition probability of that specific location and the subsequent flame behavior, see e.g. [22]. However, in summary, most of the numerical studies involved a LES-based approach and were thus limited to a very small number of calculations. As a consequence, the results only comprised a confined set of locations at which the ignitability could be elucidated and can therefore, at the current state of still restricted computational power, not be employed to yield a complete spatial map of the ignitability of a combustor. On the other hand, by means of URANS simulations based on the extended TFC model presented here, a reasonable reproduction of the ignition map of the jet flame test case studied by Ahmed et al. [16] has been achieved, see Boyde et al. [23].

Hence, in order to bridge the gap until the computational power becomes available to allow LES computations for numerous stoichiometric and air flow conditions at the igniter positions, we present a reacting multiphase flow combustion model which is applicable to a standard RANS / URANS environment. Our approach is based on the Turbulent Flame Speed Closure model, hereafter termed TFC model, which was first proposed by Zimont [24] and offers the advantage that it is of a very reasonable accuracy and robustness for premixed flames. This was successfully demonstrated by the work of Zimont et al. [25]. Its extension to non-premixed conditions was realized by Polifke et al. [26], Flohr and Pitsch [27] and Knudsen and Pitsch [28] among others. Usually, the aforementioned low-order models are optimized for a single characteristic of the combustion process; for instance certain species concentrations and pollutant emissions or ignition delay times or soot prediction. In this work, the TFC model offers the essential advantage that it captures the flame propagation speed. Precisely, the source term in the reaction progress variable transport equation is closed using a velocity scale dependent upon the physical-chemical properties of the local mixture. With the inclusion of the laminar flame speed as an intrinsic chemical property and the local turbulence quantities, a correlation is derived which approximates the propagation behavior of the flame also in laminar and turbulent regimes. Through the dependence on the laminar flame speed, detailed chemistry effects are taken into account yet retaining the simplicity of the model. The second detailed chemistry effect accounted for in our model for the reaction progress variable source term is the ignition delay time. It has been identified by Lefebvre [11] that three phases determine the success of the gas turbine light-off. Phase 1, which represents the deposition of energy to achieve a sufficiently large initial flame kernel is addressed in our ignition model extension. Phase 2, corresponding to the propagation and growth of the flame kernel into the primary zone and, Phase 3 the "light around" where flame kernels from an ignited fuel injector propagate circumferentially around the annular combustor both directly depend on the flame speed. Thus, with our focus on relight or light-off modeling, the TFC model represents a very adequate choice among the low-order combustion models.

The TFC model itself has undergone a series of enhancements since its formulation by Zimont [24]. One of the improvements made by Zimont and Lipatnikov [29] limits the turbulent flame speed needed for the source term in the presence of high turbulence. Formerly, the increase of turbulence fluctuations would also cause a rise in the absolute value of the turbulent flame speed, which is only valid for Karlovitz numbers less than one. Up to this condition, the small eddies of the size of the Kolmogorov length scale are larger than the inherent reaction zone of the flame front. Beyond that point, for Karlovitz numbers greater than one in case of stronger turbulence, the chemical reactions are affected by the small scale turbulence, which reduces and ultimately quenches the flame. A further contribution to the flexibility of the model was accomplished by Polifke et al. [26] and also Cokljat et al. [30] who proposed the transport of the mixture fraction and the sensible enthalpy in order to adapt the model to nonpremixed environments and to incorporate non-adiabatic effects. The model can also be used in the context of LES simulations, as demonstrated by Knudsen and Pitsch [28].

To the best of the authors knowledge, simulations involving the TFC model for multiphase flows are not present in the literature, nor the common model implementation apt to incorporate multiphase reactants phenomena. Thus, our contribution is the extension of the model formulation to allow the treatment of multiphase reactants in turbulent flows.

Furthermore, there are some shortfalls associated with 
the standard model implementation. A numerical study performed by Wood and Moss [31] revealed that problems arise for example in situations where a fully burnt lean mixture connects with pure air. Pure air is difficult to describe with a reaction progress variable. In the original model, $c$ corresponds to the normalized ratio of the current local product mass fraction to the value of the maximum attainable local product mass fraction: $c=Y_{p} / Y_{p, b}$. Hence, under premixed conditions $c$ is unity within the flame and zero outside the flame. The situation becomes more complex in a non-premixed scenario. Without the presence of fuel, pure air cannot react and the reaction progress variable is undefined. Thus, capturing the dilution of products from lean combustion with air constitutes a challenge. This process should not alter the reaction progress variable considering that a further chemical reaction cannot occur since only fuel is scarce. In the original model, the reaction progress variable is nevertheless reduced due to the mixing of air with a flame progress variable value of zero and combustion products with a flame progress variable value of unity. We will demonstrate that this dilemma is solved by applying the extended model formulation presented in this paper. The approach we propose, demands the transport of selected species which enables the determination of the physical reaction progress and avoids an erroneous influence of mixing effects. The improvement of a low order model by adding a second nonnormalized flame progress variable (which can be derived from the transported species in our case) to the already existing normalized one, has been successfully applied to other models previously, see e.g. Colin and Benkenida [32], Vervisch et al. [33] or by Van Oijen et al. [34]. Although this renders the model more costly in terms of computational time, we will elaborate that the advantages and the possibilities of the model far outweigh this issue. The second model improvement highlighted here is the ignition interface, which was also not available in the standard model. As we aim at using the model to study ignition problems this represents an essential and valuable extension of the model abilities.

The study is structured as follows. First, the fundamental model equations are discussed. Subsequently, the elementary validation is presented by means of three simplified test cases, a Bunsen burner flame, a counterflow flame and, a multiphase reactants 1-D channel, which allow establishing the reliability of the extended model. Then, the model predictions are compared to the outcome of a lab-scale spray burner experiment, which serves as a validation of the model capabilities for turbulent reactive multiphase flows triggered by the suggested ignition extension. The generic experiment brings insight into spray ignition and flame propagation in multiphase flows, which relates to all three phases of light-off.

\section{Model Description}

\section{Flow Solver}

The numerical simulations carried out in the scope of this study employ the CFD code THETA, developed at the DLR. The code is applicable to incompressible flows and uses unstructured grids as domain representation. For stationary calculations the SIMPLE method is utilized, as detailed in Ferziger and Peric [35]. Transient calculations are run with a projection method for the pressurevelocity coupling scheme [35]. The temporal discretization is accomplished with a three-point backward method yielding second-order accuracy. For the turbulent closure a standard $k$ - $\epsilon$ turbulence model [36] with wall functions is incorporated. The equations are discretized in a vertexcentered manner and solved by a Bi-Conjugate Gradient Stabilized (BiCGStab [37]) method. Second-order upwind and central schemes are used for convective and diffusive terms, respectively. A linear multi-grid acceleration method is utilized to solve the resulting elliptic, secondorder Poisson differential equation for the pressure correction.

\subsection{Combustion Model Equations}

For the inclusion of combustion processes, an approach based on the turbulent flame speed closure model originally developed by Zimont [24], is employed. The extended TFC model is convenient for unsteady computations, in particular, ignition studies, see Boyde et al. [38-40] as it is designed for capturing the flame propagation. Since its source term is derived from the laminar flame speed, it enables an intuitive description of the flame spreading behavior. To derive the model equations we start with the simple form of the transport equation for the reaction progress variable $c$ :

$$
\frac{\partial}{\partial t}(\rho c)+\frac{\partial}{\partial x_{k}}\left(\rho u_{k} c\right)=\frac{\partial}{\partial x_{k}}\left(\rho D_{c} \frac{\partial c}{\partial x_{k}}\right)+\dot{w}_{c} .
$$

$c$ is an independent scalar which indicates the reaction progress. A major difference to the common approach [26, $27,31]$ is that we do not assign a physical species, e.g. the normalized product mass species. The reason for that is elaborated in the further course of this paragraph. The other variables occurring in Eq. (1) are the density $(\rho)$, the diffusion coefficient $\left(D_{c}\right)$ and the source term $\left(\dot{w}_{c}\right)$. Since we consider flows of variable density we apply the Favre averaging $(\overline{\rho c}=\bar{\rho} \tilde{c})$ to Eq. (1) and obtain the following form:

$$
\frac{\partial}{\partial t}(\bar{\rho} \tilde{c})+\frac{\partial}{\partial x_{k}}\left(\bar{\rho} \tilde{u}_{k} \tilde{c}\right)=\frac{\partial}{\partial x_{k}}\left(\bar{\rho} D_{c} \frac{\partial \tilde{c}}{\partial x_{k}}\right)-\frac{\partial}{\partial x_{k}}\left(\overline{\rho u_{k}^{\prime \prime} c^{\prime \prime}}\right)+\overline{\dot{w}}_{c}
$$

The turbulent flux $\left(\overline{\rho u_{k}^{\prime \prime} c^{\prime \prime}}\right)$ is modeled with the usual gradient-diffusion assumption, see e.g. [27] and [31]. 
Hence, we expand the diffusion term of Eq. (2) and introduce the turbulent Schmidt number $\left(\mathrm{Sc}_{t}\right)$, which allows to relate the turbulent flux with the turbulent viscosity using the Boussinesq assumption [41]. It has been observed by many researchers [42-44] that a counter gradient diffusion can also occur . However, this phenomenom is neglected in our implementation as the envisaged range of the model corresponds to regimes which are characterized by high turbulent Reynolds numbers: $\operatorname{Re}_{T}=u^{\prime} l_{t} / \nu \gg 1$. Under these conditions an analysis of Veynante et al. [45] revealed that counter gradient diffusion is not the dominant direction of the turbulent flux as the large velocity fluctuations govern the flame behavior. For our validation study, associated with conditions of weak turbulence, the counter gradient diffusion is also negligible as the velocity fluctuations are of very low magnitude, which limits the impact of the turbulent flux in general. Thus, we end up with the closed transport equation for $\mathrm{c}$ :

$$
\frac{\partial}{\partial t}(\bar{\rho} \tilde{c})+\frac{\partial}{\partial x_{k}}\left(\bar{\rho} \tilde{u}_{k} \tilde{c}\right)=\frac{\partial}{\partial x_{k}}\left(\bar{\rho} \frac{\nu_{t}}{\mathrm{Sc}_{c_{t}}} \frac{\partial \tilde{c}}{\partial x_{k}}\right)+\bar{w}_{c}
$$

Note that the diffusion term only contains the contribution from the turbulent diffusion $\nu_{t}$, as the laminar diffusion part is contained intrinsically in the source term, as discussed, e.g. by Durand [46]. The expression $\mathrm{Sc}_{c_{t}}$ corresponds to the turbulent Schmidt Number of $c$, which is equal to 0.7 in all simulations.

The source term in the transport equation for the reaction progress variable is given as:

$$
\overline{\dot{w}}_{c}=\rho_{u} S_{T}|\nabla \tilde{c}|+C_{\text {ign }} \bar{\rho} \frac{1}{t_{i}}
$$

The first part of Eq. (4) relates to the standard implementation of the TFC model. It links the source term for $c$ to the local turbulent flame speed and to the gradient of the reaction progress variable. One widely employed expression and present in the original formulation for the turbulent flame speed is the one derived by Zimont et al. [24]. It has been substantiated that this expression is valid for the wrinkled-thickened flame regime, characterized by $\mathrm{Re}_{t} \gg 1$ and $\mathrm{Da}_{t} \gg 1$, which is usually the case in a gas turbine combustor. The turbulent Damköhler number is defined as: $\mathrm{Da}_{\mathrm{t}}=l_{t} / u^{\prime} / \chi_{\mathrm{u}} / S_{L}^{2}$. Beyond these regimes and especially for lean conditions, a quenching term becomes crucial which is specified by Zimont [47] and further elaborated in [26]. It ensures, that the turbulent flame speed cannot grow boundlessly for strong turbulence.

Other expressions for the turbulent flame speed exist in the literature, e.g. Eickhoff [48] and Schmid et al. [49], which, however lack the quenching term and/or the wide range of validity. As our validation with experimental data does not fulfill the conditions $\operatorname{Re}_{t} \gg 1$ and $\mathrm{Da}_{t} \gg 1$, we suggest the use of an expression which is proposed by Peters [50], which covers a larger range of turbulent regimes:

$$
S_{T}=S_{L}\left(1+\tilde{\sigma}_{t}\right)
$$

with $\tilde{\sigma}_{t}$ being defined as:

$$
\tilde{\sigma}_{t}=-\frac{0.39}{2} \frac{l_{t}}{l_{f}}+\left[\left(\frac{0.39}{2} \frac{l_{t}}{l_{f}}\right)^{2}+0.78 \frac{u^{\prime} l_{t}}{S_{L} l_{f}}\right]^{\frac{1}{2}}
$$

The laminar flame thickness $l_{f}$ is obtained from $l_{f}=$ $\frac{\nu / \mathrm{Sc}_{Y}}{S_{L}}$. Values for the laminar flame speed $\left(S_{L}\right)$ as a function of the equivalence ratio $\Phi$, the temperature and the pressure are prescribed using polynomial expressions for the corresponding fuel which are provided in the appendix. The turbulent flame speed expression proposed in the original version of Zimont [24] and the correlation from Peters [50] both converge for large values of $\mathrm{Re}_{t}$ and $\mathrm{Da}_{t}$. The quenching term introduced by Zimont [47] can be coupled to both expressions. However, it has been omitted in our study as the validation test cases do not exhibit a high level of turbulence and therefore, we refrain to state its equation. Concerning LES adaption of the TFC model, studies by Flohr and Pitch [27] and by Zhang et al. [51] demonstrate that this can be achieved with a computation of the turbulent flame speed based on turbulent quantities at subgrid scales.

The second contribution to the source term in Eq. (4): $C_{\text {ign }} \bar{\rho} \frac{1}{t_{i}}$ represents the triggering mechanism for the reaction progress variable. In the original TFC model no interface to capture ignition is present, hence, artificial assumptions are required to initiate a flame kernel front. As opposed to that, the proposed coupling of the reaction progress variable to the auto-ignition characteristics of the mixture yields a more natural and physical implementation. The additional source term $C_{\text {ign }} \bar{\rho} \frac{1}{t_{i}}$ follows the reasoning that a mixture is ignited once the condition: $\int_{t_{0}}^{t} \frac{1}{t_{i}} d t>1.0$ is fulfilled. At each time step the ignition delay time is evaluated for each grid cell depending on the local state which gives a corresponding finite rise in the flame progress variable. $C_{\text {ign }}$ is a model constant which is equal to 1.0 throughout this paper. Values for $t_{i}$ are prescribed using polynomial expressions which are provided in the appendix. In case the local conditions do not allow auto-ignition the value of $t_{i}$ is set to $1 \times 10^{6} \mathrm{~s}$ in order to render the second source term of Eq. (4) negligible.

The transport equation Eq. (3) allows a basic flame description within a homogeneous premixed environment. For a more complex flame regime involving several inflows of different composition and multiphase phenomena, the transport of individual species mass fractions $Y_{i}$ and specific enthalpy $h$ is required. Actually, through its source term given in Eq. (4), the evolution of $c$ depends on $S_{L}$ and $t_{i}$. Both are functions (see polynomials detailed below) of $\Phi$ and $T_{u}$ thus the need for the local specific enthalpy. This variable is transported (see Eq. (14)) and used to compute the temperature (Eq. (15)) which necessitates the selected species mass fractions $Y_{i}$. Hence, we propose to introduce transport equations for selected elementary species. 
$\frac{\partial}{\partial t}\left(\bar{\rho} \tilde{Y}_{i}\right)+\frac{\partial}{\partial x_{k}}\left(\bar{\rho} \tilde{u}_{k} \tilde{Y}_{i}\right)=\frac{\partial}{\partial x_{k}}\left(\bar{\rho} D_{Y} \frac{\partial \tilde{Y}}{\partial x_{k}}\right)+\overline{\dot{w}}_{Y_{i}}+\overline{\dot{\Gamma}}_{Y_{i}}$

In the present work, oxidizer, fuel and main combustion products (i.e. $\mathrm{CO}_{2}$ and $\mathrm{H}_{2} \mathrm{O}$ ) are transported. $\mathrm{N}_{2}$ is considered the inert species to fulfill the continuity. The source term $\bar{\Gamma}$ accounts for evaporation detailed elsewhere [52] for the sake of brevity, whereas $\overline{\dot{w}}_{Y_{i}}$ describes changes related to the reaction progress variable. $D_{Y}$ constitutes the constant diffusion coefficient of the species, which corresponds to:

$$
D_{Y}=\frac{\nu}{\mathrm{Sc}_{Y}}+\frac{\nu_{t}}{\mathrm{Sc}_{Y_{\mathrm{t}}}}
$$

with the constants $\mathrm{Sc}_{Y}$ and $\mathrm{Sc}_{Y_{\mathrm{t}}}$ also equal to 0.7. $\nu$ and $\nu_{t}$ represent the laminar and turbulent viscosity, respectively. The model extension is meant for configurations in which a single fuel species is present and for which a global reaction with an oxidizer can be derived in the form of:

$m C_{x} H_{y} O_{z}+\left[m \cdot\left(x+\frac{1}{4} y\right)-\frac{z}{2}\right] O_{2}=x \cdot m \cdot C O_{2}+m \cdot \frac{y}{2} H_{2} O$

Hence, transport equations are needed for all occurring species in the global reaction plus a remaining inert species. Since the interaction between evaporation phenomena and the TFC combustion model has not been found in the literature, some general considerations on the implications shall be given here. The first ambiguity concerning the reaction progress variable $c$ occurs when fuel droplets evaporate within the flame. In a lean mixture, the increase in fuel vapor concentration due to evaporating droplets should lead to a reduction in $c$, as the fuel vapor added to the gaseous phase is not consumed yet. To incorporate this process, we introduce a dependent variable, $\beta$, which is defined as the ratio of local exhaust gas, e.g. $Y_{\mathrm{CO}_{2}}$ to the maximum attainable local exhaust gas mass fraction $\left(Y_{\mathrm{CO}_{2} \max }\right)$ corresponding to a complete combustion.:

$$
\beta=\frac{Y_{\mathrm{CO}_{2}}}{Y_{\mathrm{CO}_{2 \max }}}
$$

The choice of $Y_{\mathrm{CO}_{2}}$ as marker is arbitrary, as for the underlying global reaction, any linearly dependent species is an adequate choice. The maximum mass fraction of $\mathrm{CO}_{2}$ can be obtained by solving the global reaction in the form of Eq. (9). The quantity $\beta$ is only defined for an equivalence ratio above 0.1 and is set to zero if below this threshold to avoid divergence in regions without any fuel. Contrary to $\beta$ the "reaction progress variable" $c$ determines where the flame resides $(c>0.0)$ and is unaffected by local fuel vapor sources. However, it does not give details regarding the local composition in our implementation. It can be interpreted as a flame edge marker, similar to the flame edge variable $G$ in the G-equation model, see e.g. [53]. The second ambiguity concerning the reaction progress variable constitutes the major problem of the standard TFC model. The reaction progress variable $c$ is undefined for pure air (we use $c=0$ ). As a consequence, the degree of reaction cannot be deduced when air with $c=0$ and exhaust gases with $c=1 \mathrm{mix}$.

For exhaust gases stemming from lean combustion the blending with pure air should not allow any further reaction progress. However, in the standard formulation the TFC model fails. It assumes a dilution of the flame progress variable which represents an independent variable that will therefore take on values between 0 and 1 in the mixing zone. Values of the reaction progress variable other than 0 and 1 indicate that unburnt fuel is existent and the reaction has not finished yet, see Wood and Moss [31]. This issue is overcome by the method presented in this paper, as the algorithm includes the transport of elementary species, see Eq. (7). As the fuel is transported explicitly, the value of its mass fraction is not directly linked to the value of the reaction progress variable.

The presence of fuel vapor sources explains the necessity of the second dependent flame progress variable $\beta$. $\beta$ ensures that a physically meaningful reaction can occur within the flame zone which is characterized by $c>0.0$. Through droplet evaporation within a lean flame or through blending of flows, the reaction progress variable does not describe the current chemical state, as the standard definition of $c=1$ suppresses any further combustion even if fuel and oxidizer are still present. As $\beta$ is evaluated at the beginning of each time step it reflects the proper chemical state of the reaction. Consequently, by means of comparing $\beta$ with $c$ we can deduce whether the current local reaction progress variable state does indeed correspond to the species mass fractions locally present.

The source term of each species is split into one part associated with the liquid phase evaporation $\left(\overline{\bar{\Gamma}}_{Y_{i}}\right)$ and one part associated with the combustion process. The source term related to combustion is modeled for each species that occurs in the global reaction as:

$$
\overline{\dot{w}}_{\mathrm{Y}_{i}}=\gamma \cdot\left(\overline{\dot{w}_{c}}+\overline{w_{\beta}}\right) \cdot n_{\mathrm{st}_{i}} \cdot M_{i} / \bar{M}
$$

where $n_{\text {st }_{i}}$ represents the $i$-th stoichiometric coefficient according to Eq. (9), $M_{i}$ the molar weight of the $i$-th species and $\bar{M}$ the average molar weight of the mixture. The factor $\gamma$ is derived by:

$$
\gamma=Y_{\mathrm{CO}_{2} \max } \cdot \bar{M} /\left(M_{\mathrm{CO}_{2}} n_{\mathrm{st}_{\mathrm{CO}}}\right)
$$

The expression $\dot{w}_{\beta}$ is computed as follows:

$$
\dot{w}_{\beta}=\rho \cdot \max (c-\beta, 0.0) /\left(D_{Y} / S_{L}^{2}\right)
$$

which achieves a steady consumption within a typical time scale, expressed by the term $D_{Y} / S_{L}^{2}$, of either fuel or oxidizer in case the local reaction progress variable $(c)$ is higher than the local chemical state of the combustion 
$(\beta)$. A simple correlation depending on turbulent diffusion and the laminar flame speed for the time scale suffices, in contrast to more sophisticated correlations as proposed in Ihme and Pitsch [54] and Michel et al. [55], since this only affects the burn-up of newly generated fuel vapor in the flame zone.

In order to allow non-adiabatic effects to be captured, the specific enthalpy needs to be accounted for by a separate transport equation, which reads:

$$
\frac{\partial}{\partial t}(\bar{\rho} \tilde{h})+\frac{\partial}{\partial x_{k}}\left(\bar{\rho} \tilde{u}_{k} \tilde{h}\right)=\frac{\partial}{\partial x_{k}}\left(\bar{\rho} D_{h} \frac{\partial \tilde{h}}{\partial x_{k}}\right)+\overline{\dot{w}}_{h}+\overline{\dot{\Gamma}}_{h}
$$

with $D_{h}=D_{Y}$, as the unity gas phase Lewis Number assumptions is employed. The source term $\overline{\dot{w}}_{h}$ accounts for energy sources such as the ignition energy which is described in Sec. (2.2) whereas liquid phase contributions such as the fuel droplet evaporation are covered by $\bar{\Gamma}_{h}$, see [52]. In most TFC implementations, see e.g. Polifke et al. [26] the temperature is derived from a correlation relating the adiabatic flame temperature with the reaction progress variable. However, outside the flammability limits, the adiabatic flame temperature is undefined, which requires artificial assumptions to be made. By employing (the transported) elementary species, the mixture temperature is obtained by virtue of:

$$
h(T)=\sum_{i=1}^{n} Y_{i} h_{i}=\sum_{i=1}^{n} Y_{i} \cdot\left(h_{0_{i}}+\int_{T_{0}}^{T} c_{p_{i}}(T) d T\right),
$$

where $n$ denotes the number of species. For $c_{p}$ the NASA polynomials are employed [56]. The density is subsequently derived by solving the equation-of-state for ideal gas.

Two unresolved tasks remain for the closure of the model. The laminar flame speed needs to be provided for the source term in Eq. (4) with consideration of Eq. (5). Furthermore, an expression for the ignition delay time needs to be found for the auto-ignition contribution in Eq. (4). Both quantities can be defined as a function of $p$, $T_{u}$ (unburnt gas temperature) and $\Phi$ which allows the use of polynomial expressions. The unburnt gas temperature is inferred through computing the initial conditions prior to combustion. This is achieved by reversing the global reaction as given in Eq. (9). The employed correlations are examined more thoroughly in Boyde et al. [57]. For completeness, the form of the polynomials and the coefficients for all fuels employed in this study are provided in the appendix. The validity of the polynomials ranges from $0.6<\Phi<1.5,293.15 \mathrm{~K}<T_{u}<593.15 \mathrm{~K}$ for the laminar flame speed and from $1200 \mathrm{~K}<T_{u}<1800 \mathrm{~K}$ for the auto-ignition time. For both quantities the unburnt temperature and the gaseous equivalence ratio are taken as references. The considered pressure range extends from
0.5 atm $<p<6$ atm. For temperatures rising beyond the maximum temperature of the respective correlation, the value at the maximum temperature is taken.

\subsection{Spark Representation}

Concerning the prescription of the ignition energy, a rather simple approach is utilized. A time period $\left(t_{s}\right)$ is specified during which the ignition energy is provided to the domain. In addition, a characteristic volume $\left(V_{s}\right)$ of the spark is prescribed. Both quantities are chosen in such a manner, that the energy fed to the domain equals the effective energy $\left(E_{s}\right)$ as measured in the experiment. This yields the enthalpy source term $\overline{\dot{w}}_{h}$ which is required in Eq. (14). $\overline{\dot{w}}_{h}$ has to fulfill the condition: $\int_{t_{s}} \int_{V_{s}} \rho \bar{w}_{h} \mathrm{~d} t \mathrm{~d} V=E_{s}$. The second constraint is the solver stability. In general a minimum of ten time steps is required for the disposal of the ignition energy to avoid solver instabilities. For multiphase flows with laser-induced ignition, a fraction of instantaneously vaporized fuel in the order to $10 \%$ of the liquid fuel present in the respective cell is added to the gaseous fuel mass fraction to account for shock wave phenomena. This methodology is explained in the further course of this paragraph. Note that the outline for the spark shape, duration and energy distribution given here can be regarded as a proposal for the spark representation. It is not a core part of the model which can be coupled to other more sophisticated spark models likewise. In the following a reasoning is given why such a simple approach is chosen.

The physical processes, e.g. the temperature increase, occur almost instantaneously. Due to immense heat losses caused by radiation, shock wave generation and losses at the electrodes for a spark plug, the extensive initial temperatures abate quickly [58-60]. Therefore, in a numerical simulation where the time steps are of the order of several $\mu \mathrm{s}$, the presumption that the ignition temperature has decreased to the order of typical adiabatic flame temperatures is justified. Secondly, the developing flow field structures at the edges of the ignition kernel, see e.g [60, 61], lead to enhanced mixing processes. This further mitigates the large gradients and entails a more homogeneous temperature distribution.

The employed modeling strategy is similar to widely acknowledged schemes, such as the energy deposition (ED) ignition model which has been successfully applied to forced ignition test cases by Chakraborty et al. [62] and Lacaze et al. [22, 63]. It is also in analogy to an approach proposed by Kravchik and Sher [64] and to modeling assumptions which were established within the automotive industry to treat spark discharges in conventional diesel engines [65].

In case ignition occurs in a two phase flow, the implications on the liquid phase need to be captured by the ignition model. Experimental observations reveal that the generated shock wave which results from the extreme temperatures, induces a secondary break-up of the droplets $[66,67]$. The droplets, therefore, adopt diameters which 
are far below their original diameters. This effect can be observed indirectly in the experimental measurements [68] and is investigated directly by Gebel et al. [67]. This leads to characteristic droplet sizes $(\mathcal{O}(1 \mu m))$ that vaporize rapidly in the hot environment. Characteristic time scales for these phenomena are too short with respect to the simulation time step. Therefore, it is modeled using an instantaneously prescribed increase in fuel vapor mass fraction.

A validation of the spark model for gaseous conditions is given in Boyde et al. [23]. In their study [23], the ignition probability map of a generic gaseous jet measured by Ahmed et al. [15] was successfully reproduced with the extended TFC model by simulating various distinct locations in the domain. It was pointed out that the stochastic nature of the ignition probability cannot be resolved with a URANS approach. One reason is that small scale turbulence is not resolved within this simulation approach. The second reason is, that a large part of the stochastic nature of the flow field is not resolved as the URANS simulation of the spark evolution is started using a converged RANS simulation for single phase flows. For multiphase flows, the URANS simulation begins at the injection of droplets to describe the proper transport of the generated droplet parcels. A converged RANS solution is used as starting point for the multiphase simulation. In general, it was demonstrated that zones with high ignition probability were well identified in the numerical simulation. The comparison indicates that favorable ignition zones are largely independent of the stochastic nature of the flow field and can be reasonably determined with the extended TFC model.

\subsection{Liquid Phase Modeling}

The transport of discrete particles is carried out following a Lagrangian approach. This is accomplished with the spray solver SPRAYSIM which was developed at the DLR. The turbulence-spray interaction is taken into account by using a droplet turbulence dispersion model based on the spectral reconstruction of local turbulent features [69].

The diffusion-controlled droplet evaporation model (see for example Sirignano [70]) derived with the sphericalsymmetry assumption for a mixture of discrete components is used. The model is extended to include convective evaporation by introducing semi-empirical correlations for conditions ranging from quiescent or Stokes flows with characteristic droplet-Reynolds numbers (here designated as parcel-Reynolds number) $\operatorname{Re}_{\mathrm{p}}=V_{\text {relative }} D_{\mathrm{p}} \rho_{\mathrm{g}} / \mu_{\mathrm{g}}$ close to zero in flows characterized by high $\mathrm{Re}_{p}$, for which a laminar boundary layer develops at the droplet surface. In the definition of the parcel Reynolds number the indice $\mathrm{g}$ denotes gaseous quantities and p parcel quantities. $D$ represents the diameter and $\mu$ the dynamic viscosity. This more general and more robust model is based upon the work of Abramzon and Sirignano [71]. It involves an iterative process for determining the heat transfer number $\left(B_{T}\right)$ and the Nusselt number for gas mixtures characterized by non-unity Lewis numbers. However, the multicomponentfuel and the non-unity Lewis number models for droplet evaporations are not used in the present study as we have pure liquid fuels and a unity Lewis number assumption. The computational burden associated with this process was substantially reduced by implementing lookup-tables. These concern the Prandtl and Nusselt numbers to the power of $1 / 3$ and importantly, for the logarithm function including the Spalding mass transfer number $\left(\ln \left(1+B_{M}\right)\right)$, which is computationally intensive (tables in increments of 0.005 for $\left.B_{M} \in[0,20]\right)$. Trajectories and property variations during each integration time step of computational particles (parcels) are computed using gas field property values provided by the gas flow solver. As a two-way coupling is implemented, source terms stemming from those parcels are fed back to the flow solver. The exact mathematical form of the mass, the momentum, the energy, the turbulent kinetic energy source, and the turbulent kinetic energy dissipation source terms are given elsewhere [52]. It should be noted that the pointwise approach used traditionally and here as well in Eulerian-Lagrangian methods for multiphase flows does not allow to account for heterogeneities at the droplet scale. A multiphase mixture fraction definition introduced by Bilger [72] and Mortensen \& Bilger [73] seems promising.

\section{Single Phase Validation}

This section comprises some fundamental academic test cases which are intended to validate the model behavior with the proposed enhancements. The selected test cases are of very basic nature, thus, boundary conditions or numerical schemes only exert a minimum influence on the final outcome which serves the purpose of assessing the functionality of the model.

\subsection{Bunsen Burner}

The first test case represents a Bunsen burner-like configuration as found for example in Liberman [74], by which means the laminar flame speed of a mixture can be determined. The numerical results should comply with the laminar flame speed associated with the imposed boundary conditions in terms of temperature and mixture composition. Since the outcome is uniquely determined by an analytic solution, see Peters [75], the model results can be assessed well. The numerical domain is a $10^{\circ}$ slice of an axi-symmetric Bunsen burner with the boundary conditions imposed as listed in Table 1. The grid is quasi 2-D since the number of grid cells in perpendicular direction equals two.

Concerning the inlet boundary conditions, a transition layer is introduced that slowly reduces the velocity from the inner tube to the velocity of the outer tube which improves the solver convergence. In the simulation the flame attachs approximately at the position at which the imposed inflow velocity is of the same magnitude as the 
Table 1: Boundary conditions of the Bunsen burner configuration.

\begin{tabular}{l|lllll}
\hline General & \multicolumn{5}{|l}{ Smallest grid spacing: $\Delta x=1.56 \times 10^{-4} \mathrm{~m}$} \\
& Flame thickness: $\delta_{f}=42.5 \mu \mathrm{m}$ & & & \\
\hline \multirow{3}{*}{ Inlet } & Outer Tube & $z>0.045$ & $V: 0.1 \mathrm{~m} / \mathrm{s}$ & $Y_{\mathrm{CH}_{4}}=0.0$ & $T=300 \mathrm{~K}$ \\
& Transition Layer & $0.045>z>0.03$ & $V: 0.1-0.8 \mathrm{~m} / \mathrm{s}$ & $Y_{\mathrm{CH}_{4}}=0.055$ & $T=300 \mathrm{~K}$ \\
& Inner Tube & $0.03>z>0.0$ & $V: 0.8 \mathrm{~m} / \mathrm{s}$ & $Y_{\mathrm{CH}_{4}}=0.055$ & $T=300 \mathrm{~K}$ \\
\hline Outflow & Pressure Outlet, $p=1.01325$ bar & & & \\
\hline Symmetry & Symmetry Condition on both sides & & & \\
\hline Wall & Adiabatic No-Slip Wall & & & \\
\hline \hline
\end{tabular}

laminar flame speed. Note that the flame position is not the validation criterion but rather the cone angle which is dependent upon $S_{L}$.

Three grid configurations have been utilized to test the model against grid dependency. Table 2 lists the different test cases. The outcome of each of the simulations exhibits

Table 2: Grid configurations for the Bunsen burner test case.

\begin{tabular}{cc}
\hline \hline Test Case No. & Grid Dimensions \\
\hline 1 & $161 \times 91$ Nodes \\
2 & $322 \times 182$ Nodes \\
3 & $644 \times 364$ Nodes \\
\hline \hline
\end{tabular}

the same features as the ones displayed in Fig. 1.

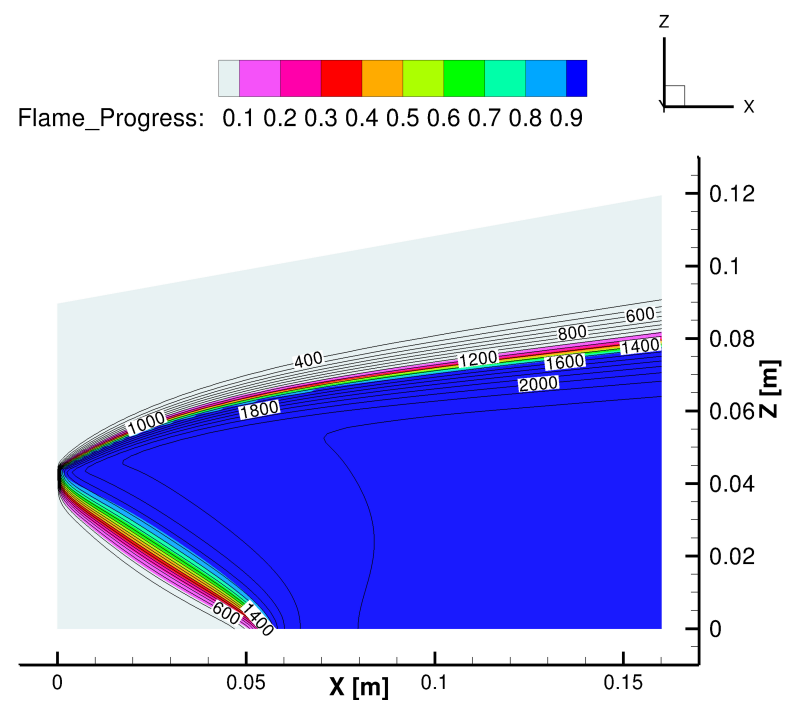

Figure 1: Contour plot of the reaction progress variable for the Bunsen burner configuration. The iso-lines relate to the computed temperature.

At the central inflow the flame cannot attach as the prevailing mean flow velocity exceeds the laminar flame speed. Consequently, a Bunsen cone develops which allows a derivation of the laminar flame speed of the mixture. The relationship between laminar flame speed and Bunsen exit velocity reads:

$$
S_{\mathrm{L}, u}=v_{u} \sin \alpha .
$$

Table 3: Bunsen burner angle of the corresponding grid resolution.

\begin{tabular}{ccc}
\hline \hline Test Case No. & Computed Angle & $S_{L, u}$ \\
\hline 1 & $33.6^{\circ}$ & $0.443 \mathrm{~m} / \mathrm{s}$ \\
2 & $31.2^{\circ}$ & $0.414 \mathrm{~m} / \mathrm{s}$ \\
3 & $31.2^{\circ}$ & $0.414 \mathrm{~m} / \mathrm{s}$ \\
\hline \hline
\end{tabular}

$S_{\mathrm{L}, u}$ represent the laminar flame speed of the unburnt mixture and $\alpha$ denotes the opening angle of the Bunsen cone. For an inlet velocity of $0.8 \mathrm{~m} / \mathrm{s}$ and a stoichiometric methane/air mixture at $300 \mathrm{~K}$, which has a laminar flame speed of $\sim 0.41 \mathrm{~m} / \mathrm{s}$, the analytically derived angle equals $31^{\circ}$. The resulting angle in the simulation has been calculated by employing the following procedure:

- Post-process data file and extract lowest position with respect to $z$ at which the reaction progress $c=0.05$.

- Regress $x, z$ data of these data points with a linear function.

- Slope of the linear function corresponds to the angle of the Bunsen burner cone.

The procedure has been applied to all three grid configuration with the results for the cone angle and the corresponding laminar flame speed provided in Table 3.

For all grids a very satisfying agreement with the theoretical cone angle value is achieved. Moreover, it was demonstrated, that the model shows a good robustness to grid variations and can pertain the high accuracy on coarse grids as well. The second aspect underlined is the good capability of the model to capture flame propagation characteristics. Simulations with different equivalence ratios showed a similar good agreement, however are not presented here for conciseness.

Additionally, the extended model does not suffer from spurious sharp changes which evolve in case only the mixture fraction is transported. Actually, the reaction progress variable drops rapidly to zero at locations where the mixture composition approaches the lower flammability limit as a result of the laminar flame speed approaching zero. This is perceivable in Fig. 1, which illustrates the flame progress variable contour. In stationary computations an abrupt change in the reaction progress variable 
will persist in these regions as the flame progress variable is not subject to diffusion. This entails a very steep nonphysical temperature decline, which originates from the fact that in the original TFC model the temperature is directly coupled to the mixture fraction and to the reaction progress variable, see e.g. Polifke et al. [26]. As a consequence, a very steep temperature gradient will occur in the original model at the location of the flame progress variable jump.

\subsection{Counter-flow Configuration}

The second test case comprises a counter-flow flame configuration. This shall demonstrate the correct mixing behavior in case a burnt and an unburnt flow mix. In the original TFC model [24], some physical inconsistencies were present as explained in Sec. (2.1), which are not observed here with the improved formulation. The setup of the configuration and the boundary conditions are provided in Fig. 2 and Table 4, respectively.

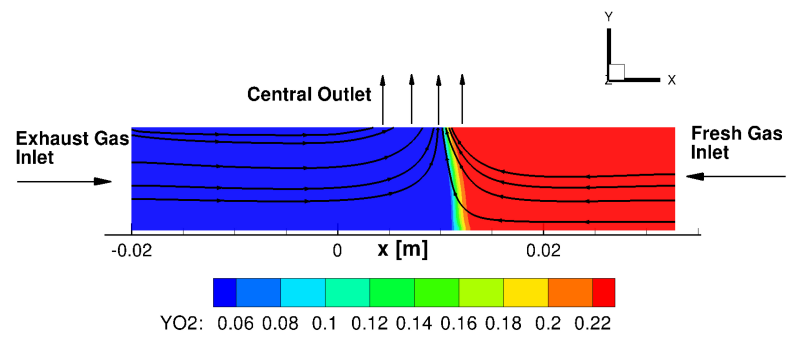

Figure 2: Set-up of the counter-flow test case. A contour plot of the mass fraction of $\mathrm{O}_{2}$ is presented. Stream-lines indicate the direction of the flow. The left inlet provides hot exhaust gases stemming from a lean combustion which mix with the fresh air flowing in from the right.

In this set-up, a fully burnt methane/air mixture with an equivalence ratio of $\Phi=0.7$ enters from the left. Fresh air is provided from the right. The inlet velocities have been chosen so that the impulse of both flows is of roughly the same order. The pressure outflow at the top is located in between $x=0.0 \mathrm{~m}$ and $x=0.0127 \mathrm{~m}$. Our intention is to demonstrate the proper mixture behavior of the proposed model. To this end, the test case has been calculated a second time with a detailed mechanism and a finite rate chemistry (FRC) scheme on the same grid. The finite rate chemistry scheme accomplishes a very accurate solution for this kind of set-up as the flow regime remains laminar. The GRI 3.0 mechanism [76] has been employed for the comparison. Note that a reduction in computational time up to a factor of 20 is achieved when using the extended TFC model as compared to the FRC model. However, due to the fact that the extended TFC model employs species mass fraction transport equations, the computational effort in comparison to the standard TFC model approximately doubles. Both results, for the present TFC model extension and the FRC model with respect to the predicted temperature are presented in Fig. 3 in addition to a third calculation corresponding to the standard TFC model. As opposed to the present TFC model extension, where selected single species are transported, in the standard TFC implementation the mixture fraction is transported. The temperature is then determined using a polynomial of the adiabatic flame temperature for a given mixture fraction and reaction progress variable. The polynomials are taken from Müller et al. [77].

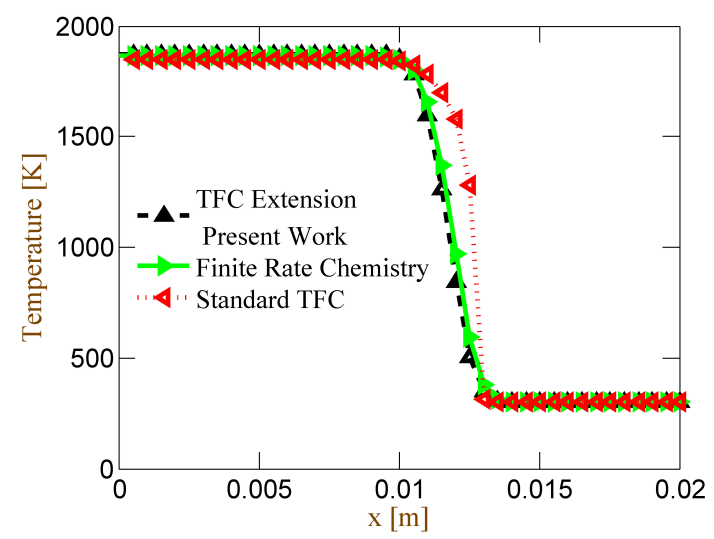

(a)

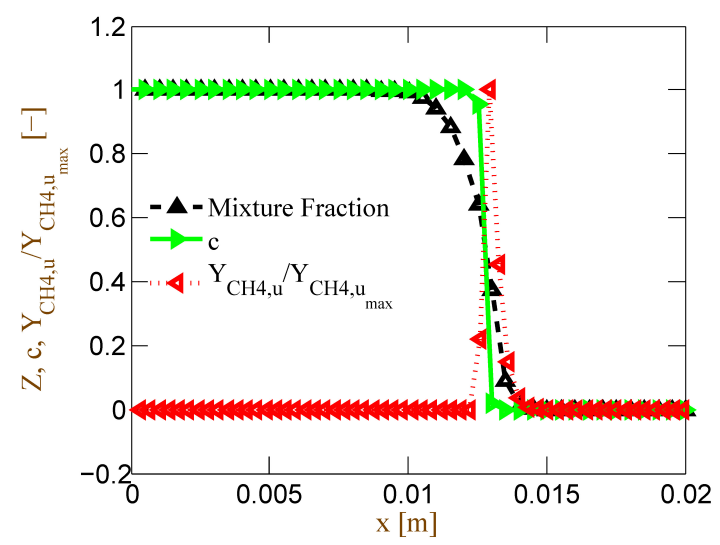

(b)

Figure 3: a) Model comparison for the axis temperature profiles of the laminar counter-flow configuration. b) Central axis profiles for the standard TFC model. Note, the unphysical generation of fuel in the standard TFC model. This raise does not occur with the present TFC model extension.

The temperature profiles from the present TFC model extension show a very good agreement with the values derived from the detailed mechanism as depicted in Fig. 3a. Slight deviations relate to a different equilibrium temperature derived in the extended TFC model. Since the present TFC model extension does not incorporate, for example $\mathrm{CO}$ as a major species, which has an impact on the equilibrium temperature, the temperature in general is slightly overpredicted. However, as the TFC model belongs to the low-order models, limitations cannot be completely avoided.

In contrast to the new implementation, the plot in Fig. $3 \mathrm{~b}$ shows the fuel mass fraction in the standard model, 
Table 4: Boundary conditions of the counter-flow test case.

\begin{tabular}{|c|c|c|c|c|}
\hline General & $\begin{array}{l}\sim 40.000 \text { Grid Points, } 5^{\circ} \text { cake pie. } \\
\text { Smallest grid spacing: } \Delta x=1.5 \times 10^{-6} \mathrm{~m} .\end{array}$ & & & \\
\hline Left inlet & Temperature $1872 \mathrm{~K}$ & Velocity: $2.1 \mathrm{~m} / \mathrm{s}$ & $\begin{array}{l}Y_{\mathrm{H}_{2} \mathrm{O}}=0.1207 \\
Y_{\mathrm{CH}_{4}}=0.0266\end{array}$ & $\begin{array}{l}Y_{\mathrm{CO}_{2}}=0.1474 \\
Y_{\mathrm{N}_{2}}=0.70534\end{array}$ \\
\hline Right inlet & Temperature $300 \mathrm{~K}$ & Velocity: $-0.3 \mathrm{~m} / \mathrm{s} \quad c=0$ & $Y_{\mathrm{O}_{2}}=0.23$ & $Y_{\mathrm{N}_{2}}=0.77$ \\
\hline Out Flow & $\begin{array}{l}\text { Pressure Outlet ( } p=1.01325 \text { bar }) \text { in the } \\
\text { wall. Walls are modeled as adiabatic no sl}\end{array}$ & $\begin{array}{l}\text { entral section of the upper } \\
\text { boundary condition. }\end{array}$ & & \\
\hline
\end{tabular}

obtained by means of the mixture fraction variable and the reaction progress variable. Fuel is created behind the mixing zone since in this region, the mixture fraction is greater than zero in conjunction with a reaction progress variable less than one.

In summary, the new model implementation resolves the known issues with mixing of burnt gases with a flow of a different composition. This stems from the substitution of the mixture fraction variable with selected independent species. Consequently, the extended model predicts the correct diffusion of burnt gas into fresh air.

\section{Multiphase Flow Validation}

\subsection{D Planar Flame}

The dedicated test case represents a very simple configuration of a 1D channel with an inflow at the left hand side and an outflow at the opposite end. The confinement is modeled as symmetry boundary conditions. In general, the flame speeds, which evolve in such a configuration are quite different from those of pure gaseous flames, which is associated with the occurring evaporation processes. This can cause, in case of a high overall (liquid + gas) equivalence ratio, rather beneficial conditions for the flame to propagate as the fuel may slowly evaporate and lead to stoichiometric conditions in the gas phase within a narrow region. On the other hand, an overall equivalence ratio of 1.0 leads to a more distributed combustion as the fuel droplets evaporate within a longer part of the channel. This causes the flame to propagate slower, since the gradients in the flame are not as strong as for the high overall equivalence ratio. This is what was also observed by the numerical study of Neophytou and Mastorakos [78], who applied a detailed chemistry scheme to obtain flame speed results in this configuration. They also excluded single droplets effects which may impact the outcome, since droplet surrounding flames can have a local accelerating effect.

The boundary conditions are provided in Table 5. After the downstream end of the channel is occupied by a steady number of droplets, an ignition is accomplished at $\mathrm{x}=0.0075 \mathrm{~m}$ with a radius of $0.0025 \mathrm{~m}$ and a total ignition energy of $30.0 \mathrm{~mJ}$. Subsequently, a flame kernel develops in the ignition zone. After the ignition effects have abated $(t>10 \mathrm{~ms})$, the flame propagation can be measured. The flame front is tracked and dividing the spatial displacement by the passed time yields a value for the laminar flame speed. The investigation has been carried out for n-decane as liquid fuel, for a set of equivalence ratios and droplet diameters. A comparison to the study of Neophytou is presented in Fig. 4.

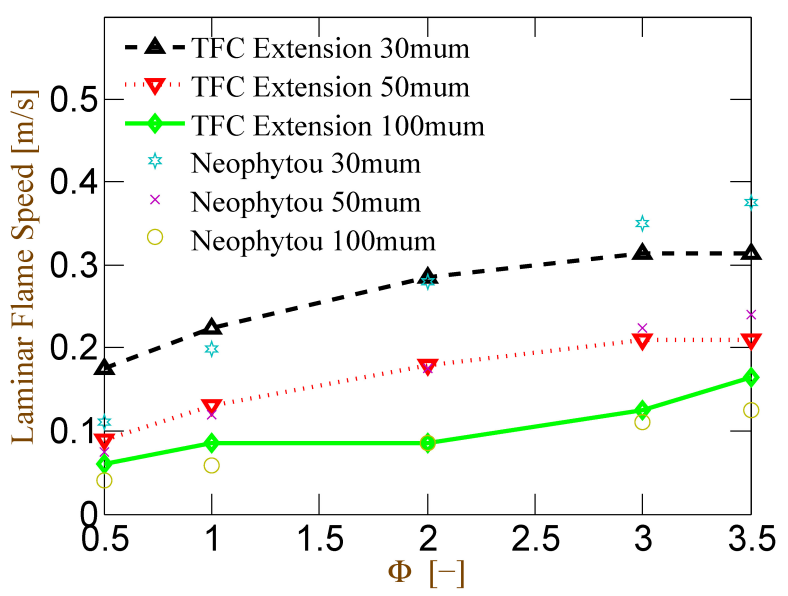

Figure 4: Comparison with numerical results from Neophytou and Mastorakos [78] for predictions of the laminar flame speed for different droplet sizes and equivalence ratios.

In summary, the model has demonstrated, that it is capable of offering a reliable prediction for the propagation of a planar flame in a multiphase flow environment. A good agreement can be achieved for this case of a simplified propagating laminar flame due to two reasons. First, the fine grid which is employed captures the flame front reasonably well. Secondly, the high resolution also enhances the accuracy of the thermal diffusion calculation which enables a proper prediction of the heat and mass source terms from the evaporating fuel droplets. Two-way coupling applied in the framework of the present EulerianLagrangian simulation ensures that the vaporized fuel vapor mass fraction is distance-proportionally interpolated to the Eulerian nodes. This is essential as the computation of the laminar flame speed includes the gaseous fuel to air ratio as one parameter in the polynomials. One drawback appears in contrast to the results obtained by Neophytou and Mastorakos [78]. They found that preferential diffusion in conjunction with a detailed chemistry scheme, can cause a laminar flame speed higher than the laminar flame speed at stoichiometric conditions. This contribution of 
Table 5: Boundary conditions of the 1D multiphase flow channel configuration.

\begin{tabular}{ll}
\hline \hline General & 1.000 Grid Points, Quasi 1D (2 Grid points in y,z direction) \\
& Smallest grid spacing: $\Delta x=1.56 \times 10^{-4} \mathrm{~m}$ \\
& Droplet diameter: $30 \mu \mathrm{m} \leq D_{d} \leq 100 \mu \mathrm{m}$. \\
& Droplet parcel rate: $5 \times 10^{5}$ parcels $/ \mathrm{s}$. \\
\hline Inlet & Temperature $300 \mathrm{~K}, Y_{\mathrm{O}_{2}}=0.23, Y_{\mathrm{N}_{2}}=0.77$ \\
\hline Spray Inlet at $x=0.02 \mathrm{~m}$ & Temperature $300 \mathrm{~K}$, Fuel: $\mathrm{n}$-decane. \\
\hline Out Flow & Pressure Outlet \\
\hline \hline
\end{tabular}

finite rate effects cannot be resolved with the simplified chemistry approach incorporated in the TFC model formulation.

\subsection{Spray Combustor}

To show the potential of the extended TFC model for technical relevant test cases, a rectangular combustion chamber fitted with a Delavan spray nozzle, which belongs to the air assisted pressure atomizer nozzle type is simulated. Experimental results obtained by Mosbach [79] allow us to compare the outcome of the numerical results for the flame development which depends on droplet size and velocity distributions and on the spray cone angle. The geometry which has a significant impact on the flow field remains of a concise design to enhance the comparison. Characteristics of the emerging spray were determined in the experiment allowing the numerical approach to be adopted to the correct boundary conditions, which is a crucial requirement to assess the performance of the combustion model.

The experimental data from Mosbach [79] offers two major objects for comparison. The first one relates to the general characteristics of the flame in terms of flame position and flame growth, provided that the ignition was successful. The second part of data comprises measurements aiming at determining the ignition probability of the set-up. For this purpose, different boundary conditions were prescribed with the intention of quantifying the influence of those on the probability of generating a fully developed flame. Accurate and repeatable energy deposition was performed using a focused laser. The amount of energy of the laser was therefore kept constant which allowed to trigger the ignition procedure several times and extract the number of successful ignition in correlation to the number of total ignitions. A further aspect was the location of the laser focus. The chance of generating a self-sustained flame is also largely dependent on the exact position where the energy is deposited. Both independent experimental results were compared to the outcome of the simulations. It is demonstrated that the agreement for both, the flame kernel development and the prediction of the ignitability is very satisfying which underlines the usefulness of the extended TFC model.

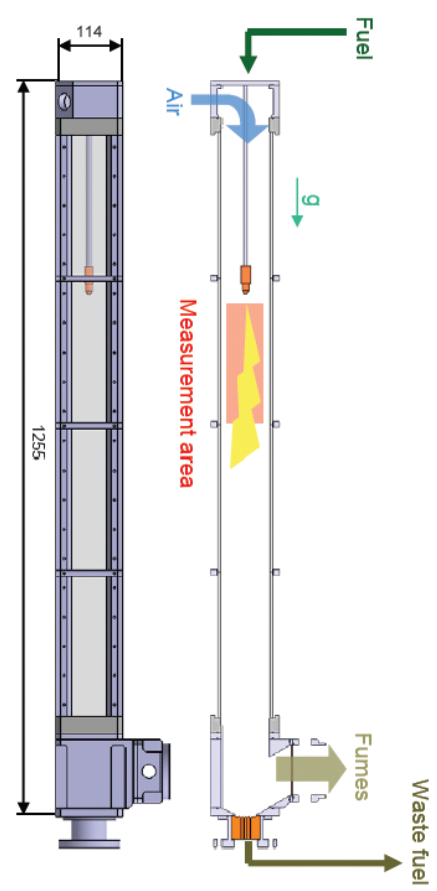

Figure 5: Sketch of the domain used in the experiment from Mosbach [79]. Air entering from the top with the fuel droplets emerging from the injector in the upper half of the channel. The laser ignition zone is located at a displacement of $4 \mathrm{~mm}$ to the middle axis.

\subsubsection{Experimental Set-Up}

The numerical examination is carried out on a grid resembling the experimentally investigated configuration of a rectangular flow channel as depicted in Fig. 5. The channel has a cross section of $6200 \mathrm{~mm}^{2}$ and a length of $1 \mathrm{~m}$. In the course of the simulations the channel length is trimmed to $500 \mathrm{~mm}$ as below approximately $450 \mathrm{~mm}$ no experimental data was taken. With this unambiguous design, the intention was to minimize the influences stemming from the geometry.

A fuel injector is embedded in the middle of the channel extending from the channel inlet to $320 \mathrm{~mm}$ downstream the axis. From the fuel injector, which corresponds to a Delavan Spray Technologies nozzle (model SN 30609-2), a spray with droplet sizes ranging from 20 to $50 \mu \mathrm{m}$ exits with velocities ranging from 5 to $40 \mathrm{~m} / \mathrm{s}$. 
Table 6: General numerical boundary conditions of the spray combustor test case.

\begin{tabular}{ll}
\hline \hline General & Smallest grid spacing: $\Delta x=0.25 \mathrm{~mm}$. \\
& Droplet parcel rate: $1.5 \times 10^{6}$ parcels $/ \mathrm{s}$. \\
\hline Air Inflow & Bulk velocity profile. \\
& Temperature $293.15 \mathrm{~K}$. \\
\hline Fuel Injector & Location: $x=0.32 \mathrm{~m} . r=8.5 \times 10^{-4} \mathrm{~m}$. \\
& Sinusoidal velocity distribution (maximum on the axis). \\
& Linear mass distribution $(0.0$ on axis, maximum at outer radius $r$ of the nozzle). \\
& Temperature: $293.15 \mathrm{~K}$. \\
& Sauter mean diameter: $35 \mu \mathrm{m}$. \\
& Root normal distribution of droplet sizes. \\
\hline Side Walls & Adiabatic no slip boundaries. \\
\hline Outlet & Pressure outlet, $p=1.01325$ bar \\
\hline Fuel & Experiment: Exxsol D80 / Simulation: n-decane. \\
\hline \hline
\end{tabular}

Table 7: Test case specific boundary conditions of the spray combustor test case.

\begin{tabular}{lll}
\hline \hline Test Case Identifier & \multicolumn{1}{c}{$\mathrm{C} 1$} & \multicolumn{1}{c}{$\mathrm{C} 2$} \\
\hline \multirow{3}{*}{ Boundary } & Air mass flow: $20 \mathrm{~m}^{3} / \mathrm{h}$ & Air mass flow: $40 \mathrm{~m}^{3} / \mathrm{h}$ \\
conditions & $v_{\text {air }}=0.96 \mathrm{~m} / \mathrm{s}$ & $v_{\text {air }}=1.92 \mathrm{~m} / \mathrm{s}$ \\
& Fuel mass flow for $6 \mathrm{SLM}$ assisting air flow & Fuel mass flow for 15 SLM assisting air \\
& in the nozzle: $17.0 \mathrm{~g} / \mathrm{min}$ & flow in the nozzle: $17.82 \mathrm{~g} / \mathrm{min}$ \\
& $v_{\text {axis }}=23 \mathrm{~m} / \mathrm{s}, v_{\text {edge }}=5 \mathrm{~m} / \mathrm{s}$ & $v_{\text {axis }}=35 \mathrm{~m} / \mathrm{s}, v_{\text {edge }}=15 \mathrm{~m} / \mathrm{s}$ \\
& Half spray angle: $19.5^{\circ}$ & Half spray angle: $16.75^{\circ}$ \\
\hline \hline
\end{tabular}

The employed fuels were Jet A-1 and Exxsol D80, a low aromatic blend of kerosene. The spray nozzle belongs to the air-assisted atomizing type, meaning that the air and fuel mix internally. By a slightly swirled flow within the nozzle, the fuel is atomized and a solid cone spray pattern with a fine droplet size distribution is produced. The air volume flow rate can be varied, ranging from $20 \mathrm{~m}^{3} / \mathrm{h}$ to $80 \mathrm{~m}^{3} / \mathrm{h}$, whereas the fuel mass flow can be prescribed from $15 \mathrm{~g} / \mathrm{min}$ up to over $35 \mathrm{~g} / \mathrm{min}$. The ignition is carried out with a frequency-doubled Nd:YAG laser which is able to generate pulse energies up to 400 $\mathrm{mJ}$ in a single shot mode. The laser focal point is located at $20 \mathrm{~mm}$ below the injector tip with a displacement of 4 $\mathrm{mm}$ with respect to the center axis.

\subsubsection{Numerical Grid \& Boundary Conditions}

Small grid cells in the ignition zone constitute one major requirement for being able to accurately define the laser pulse ignition location. However, the modeling requirement due to the presence of a dispersed phase imposes grid cells larger than droplet sizes, which opposes the former condition related to the ignition kernel. For this reason the grid cells in the part just below the injector are of adequate small size of about $0.25 \mathrm{~mm}$ for the smallest edge length, representing a compromise between the two demands. As the inlet velocity at the beginning of the channel ranges from $0.96 \mathrm{~m} / \mathrm{s}$ to $3.95 \mathrm{~m} / \mathrm{s}$, the coarse discretization at the top of the channel is justified. In total, the number of cells is of the order of 680,000 . This enables an efficient calculation with a URANS approach with the main features being well resolved. Regarding grid independency, a former study conducted on a similar grid, see [80] has pointed out that the chosen grid resolution is of appropriate quality and changes to the grid do not affect the solution. For this study, the number of computational parcels injected into the domain has been varied to analyze the influence on the simulation results. The standard value for the number of particles per second has been set to: $1.5 \times 10^{6}$ [parcels/s]. An increase of this value by a factor of four has not changed the outcome by more than $4 \%$ regarding the flame center and edge locations. The prescribed boundary conditions for the numerical domain can be found in Table 6 . The specific boundary conditions such as the mass flows and the spray cone angle concerning the respective test cases are provided in Table 7 . Note that, although it is referred to an assisting air co-flow in the nozzle, only its impact on the droplet characteristics as stated in Table 7 is modeled. It is assumed that the influence of the additional air mass $(\sim 1-2 \%$ total gaseous mass) concerning the gas phase flow field is negligible.

\subsubsection{Results}

First, a cold flow comparison is presented which is split into a single phase part and a second part in which the spray velocities are compared to the experimental data. After establishing the flow field agreement, the simulation results including combustion are analyzed with the experimental outcome with regard to flame position and flame extension. 


\section{Cold Flow}

In the experiment, a one sided inlet with a downstream sinter plate was prescribed. For the computations, this rather complex boundary condition has been simplified. The flow field without droplets is only of minor importance. Later results point out that the flow field downstream of the injector in which the flame establishes is dominated by the liquid phase velocity. The results for the single phase flow field are depicted in Fig. 6a. Discrepancies in the flow field occur in the prediction of the recirculation zone, which is overestimated by the simulation. This originates from a more stable inflow boundary condition in the simulation, which promotes the establishment of a larger recirculation zone. In the experiment, the recirculation zone is shortened, due to coherent structures which develop through the asymmetric inflow. However, the general agreement is still satisfying and should not impede the reactive simulation.

PIV measurements have been accomplished in the course of the experiment. The data can be used to compare the spray velocities obtained from the particle solver. Generally, the velocity distribution is well reproduced, as illustrated in Fig. 6c. The derived droplet velocities from registration planes in the simulation exhibit the same features as the experimental curves. The droplet velocity on the axis amounts to $\sim 28 \mathrm{~m} / \mathrm{s}$, being the maximum observed velocity and declining to $\sim 7 \mathrm{~m} / \mathrm{s}$ at the spray cone edge. The spray cone half-angle of $\sim 16.75^{\circ}$ remains nearly constant within the first $20 \mathrm{~mm}$, supporting the assumption that the flow field is dominated by the liquid phase and hence no large deformation of the spray pattern occurs. In summary, the spray and gas flow velocity boundary conditions prior to ignition are close to the experiment which allows an adequate comparison of the results for the reactive simulation.

\section{Ignition Kernel Development}

In the experiment the ignition of the spray was carried out with a laser-induced breakdown. As argued before, the proper modeling of the laser ignition can be achieved with the rather basic assumptions of a heat source and an initial vapor fraction. The ignition in the following is modeled phenomenologically by providing an energy source of $25 \mathrm{~mJ}$ over a period of $0.2 \mathrm{~ms}$ in a spherical volume with a radius equal to $2.0 \mathrm{~mm}$. The time scale in which the energy is provided is certainly larger than that in the experiments, yet the absolute value of the effectively transferred energy to the surrounding gas corresponds to the experimental set-up. In addition, a fraction of $10 \%$ of the liquid fuel mass contained in the ignition volume (sphere with radius $=2.0 \mathrm{~mm}$ ) is prescribed to be instantaneously vaporized. An almost instantaneous vaporization is also observed in the experiment as a shock wave induced by the laser breakdown causes a secondary break-up [67]. This leads to very small time scales for the evaporation. Once the ignition energy is provided,

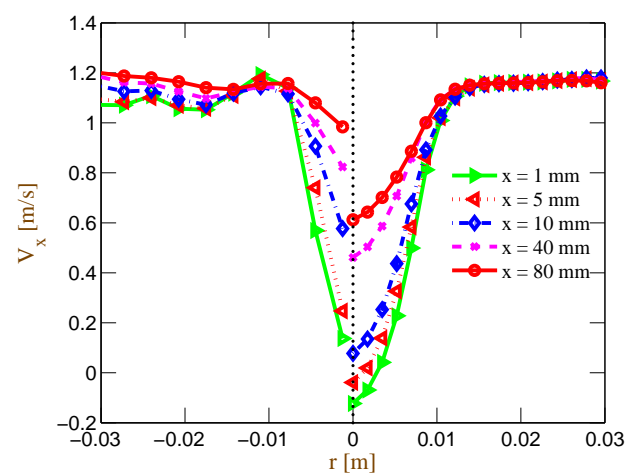

(a)

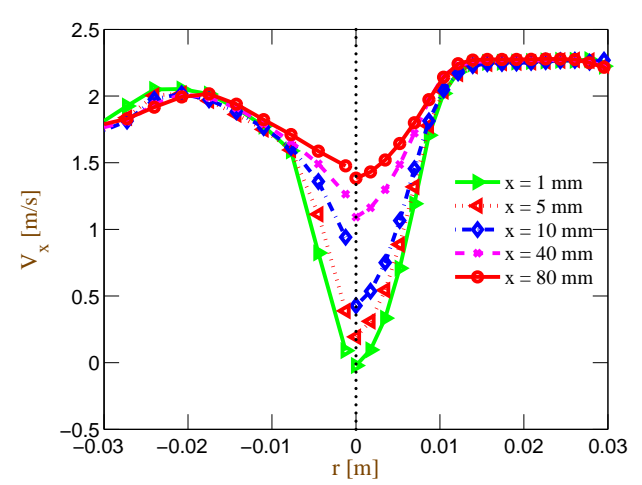

(b)

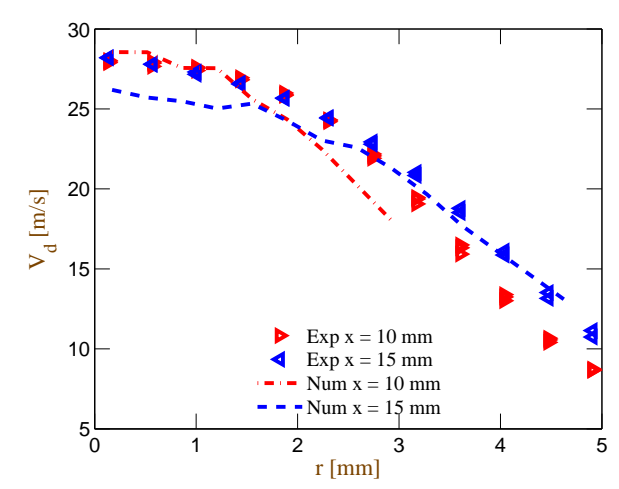

(c)

Figure 6: a) Results for the C1 case: Comparison of measured velocities (left half) and simulated velocities (right half) for the axial velocity of the single phase flow. b) Results for the C2 case: Comparison of measured velocities (left half) and simulated velocities (right half) for the axial velocity of the single phase flow. c) Results for the C2 case: Droplet velocities in the experiment and simulation

a first ignition kernel establishes, which is fueled by the initial vapor. The temporal development of the flame is depicted in Fig. 7 which also contains a qualitative comparison with experimental data. The iso contour of $1000 \mathrm{~K}$ is selected as basis for the comparison with images of the $\mathrm{OH}$-chemiluminescence since this temperature approximately equals the inner-layer temperature of the 
flame.
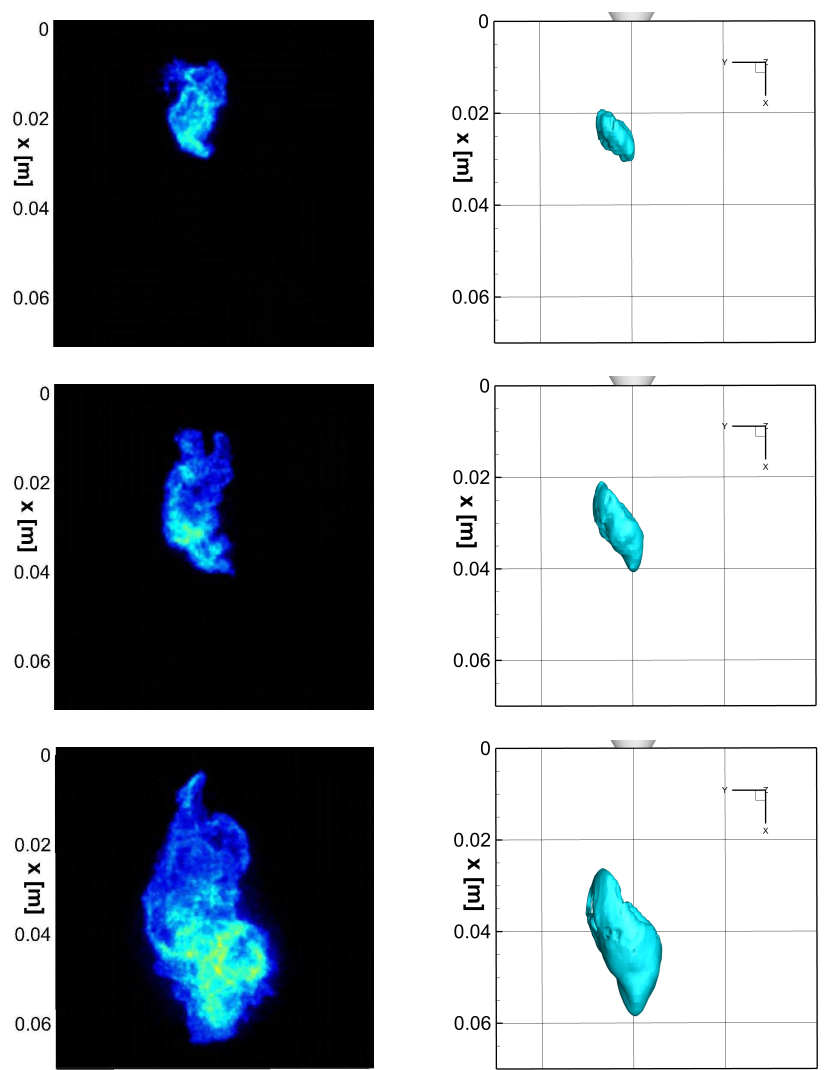

Figure 7: Results for the C2 case. Qualitative comparison of flame shape (Left: Experiment OH-chemiluminescence distribution; Right: Simulation (Iso Surface of $1000 \mathrm{~K}$ )) for three different time instances. 1st row: $1 \mathrm{~ms}$. 2nd row: 2 ms. 3rd row: $4 \mathrm{~ms}$.

To establish a quantitative assessment, general features, such as the flame center position and the flame edge location as a function of time have been recorded. For this purpose, volume averages of the $\mathrm{OH}$-Chemiluminescence were evaluated to determine the flame center position. For the flame edges, a linear method was utilized to infer the position. The same methods have been applied to the data from the numerical simulation. This allows a one to one comparison with experimental data which is presented in Table 8 and Fig. 8 .

The flame center propagation was captured very accurately. Since the convection of the flame itself is governed by flow characteristics, this is more easily computed by the numerics than the flame edge propagation which is more significantly influenced by chemistry. The former evaluation of the flow field has pointed out that the overall agreement is very satisfying which results in a sound description of the flame location. Regarding the flame edge speed, some slight deviations occur, which can be attributed to the spray characteristics. These are not contained in all detail in the experimental data, as for example the Sauter mean diameter has not been mea-

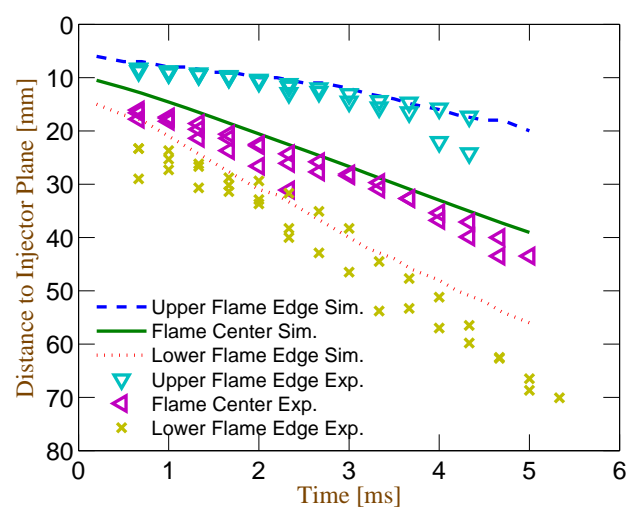

Figure 8: Results for the C2 case. Flame edges and center positions in comparison between experiment and simulation.

sured. A mean value is given by the manufacturer which has been used as boundary condition in the simulation. The results, which were presented for the first multiphase test case in this paper, confirm that in droplet mists under rich conditions smaller droplet diameter increase the laminar flame speed. In addition, the fuel used in the experiment corresponds to a blend of kerosene. As kerosene also exhibits some more volatile components, the treatment of the liquid fuel as a single component fuel ndecane can cause a deceleration of the flame front as well.

\subsubsection{Ignition Map}

The good agreement for the flame propagation characteristics indicates that the chosen assumptions are adequate to simulate the ignition kernel development. Furthermore, velocity fields were fairly well to well matched as was illustrated in Fig. 6. One aspect which has not been fully contemplated so far is the envelope of the ignition with respect to location and imposed boundary conditions. Both are important factors in a real combustor for the success of an ignition sequence. In this paragraph the capability of the model to support the determination of favorable conditions for the generation of a viable flame kernel in a spray environment is elaborated.

Table 9: Test case specific boundary conditions for a variation of the assisting air mass flow in the nozzle. SLM stands for Standard Liter per Minute. $V_{d, \max }$ represents the magnitude of the droplet velocity on the centerline and $V_{d, \text { min }}$ the velocity at the outer radius of the fuel nozzle. A linear distribution is prescribed in-between.

\begin{tabular}{lccc}
\hline \hline Quantity & 10 SLM & 15 SLM & 20 SLM \\
\hline$\dot{m}_{\text {fuel }}\left[10^{-3} \mathrm{~g} / \mathrm{min}\right]$ & 18.56 & 17.82 & 17.08 \\
Half Cone Angle [ $\left.{ }^{\circ}\right]$ & 19.5 & 16.75 & 15.6 \\
$V_{d, \max }[\mathrm{m} / \mathrm{s}]$ & 28.0 & 35.0 & 40.0 \\
$V_{d, \min }[\mathrm{m} / \mathrm{s}]$ & 10.0 & 15.0 & 20.0 \\
\hline \hline
\end{tabular}


Table 8: Comparison of numerical and experimental results of the flame characteristics.

\begin{tabular}{lccc}
\hline \hline & Flame Center Speed & $\begin{array}{c}\text { Upper Flame Edge } \\
\text { Speed }\end{array}$ & $\begin{array}{c}\text { Lower Flame Edge } \\
\text { Speed }\end{array}$ \\
\hline C1 Case & & & \\
Experimental Results & $3.62 \pm 0.13 \mathrm{~m} / \mathrm{s}$ & $1.01 \pm 0.07 \mathrm{~m} / \mathrm{s}$ & $6.24 \pm 0.19 \mathrm{~m} / \mathrm{s}$ \\
Numerical Results & $3.81 \mathrm{~m} / \mathrm{s}$ & $2.32 \mathrm{~m} / \mathrm{s}$ & $5.45 \mathrm{~m} / \mathrm{s}$ \\
\hline C2 Case & & & $10.08 \pm 0.40 \mathrm{~m} / \mathrm{s}$ \\
Experimental Results & $6.04 \pm 0.23 \mathrm{~m} / \mathrm{s}$ & $5.26 \pm 0.69 \mathrm{~m} / \mathrm{s}$ & $9.19 \mathrm{~m} / \mathrm{s}$ \\
Numerical Results & $6.43 \mathrm{~m} / \mathrm{s}$ & $3.5 \mathrm{~m} / \mathrm{s}$ & \\
\hline \hline
\end{tabular}

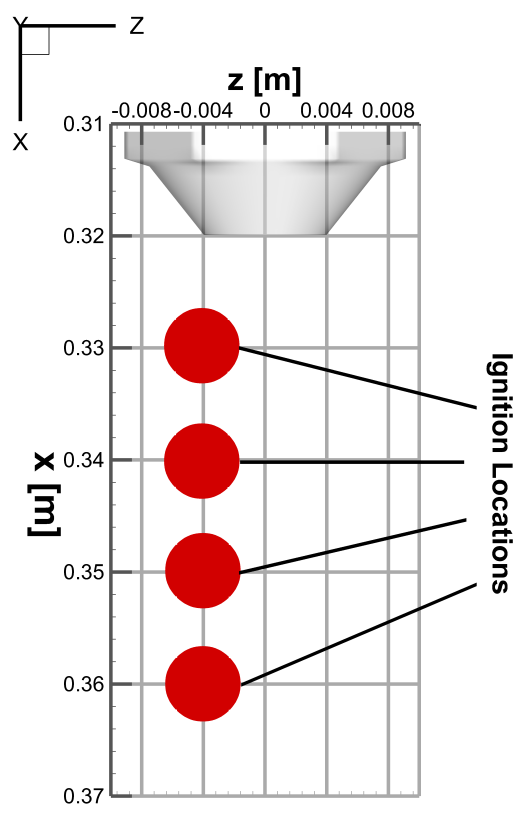

Figure 9: Investigated ignition locations. The cross-axis displacement is kept constant at $z=-4 \mathrm{~mm}$. For the axis location four distinct distances to the nozzle exit plane are chosen: $x=1 \mathrm{~cm}, 2 \mathrm{~cm}, 3 \mathrm{~cm}$ and, $4 \mathrm{~cm}$.

In this ignition study, two variations have been studied. One is the difference of ignitability with respect to the prescribed boundary conditions of the fuel nozzle. The different settings which have been investigated are listed in Table 9. The other is the impact of the location of the energy source on the overall outcome of the ignition event. The investigated ignition locations are presented in Fig. 9.

It is found that the ignition is very sensitive to the initial conditions which surround the location where the ignition energy is released, especially for the ignition with a distance of $1 \mathrm{~cm}$ to the fuel nozzle exit plane. There, the prevailing conditions have a significant impact. No successful flame is generated if the ignition kernel is produced too far off of the spray cone edge, as the very limited amount of available fuel droplets does not sustain the flame. Concerning different locations well inside the spray cone, major differences between the last successful ignition

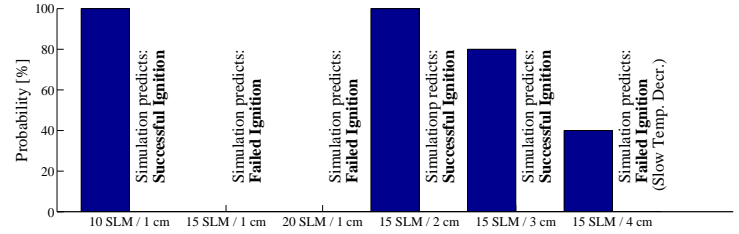

Figure 10: Ignition probability measured in the experiment (bars) and a comparison to the results from the simulation.

location at $3 \mathrm{~cm}$ and the partially successful ignition location at $4 \mathrm{~cm}$ are observed. This is due to local relative velocities between the gas phase and the liquid phase. In case of an energy deposition $4 \mathrm{~cm}$ below the injector plane it prevents the intrusion of new fuel droplets and the exit of heated up droplets in and from the flame. For locations further upstream, the local relative velocities are higher, which allows droplets to leave the flame and transport fuel vapor to the vicinity of the flame kernel. A comparison of the experimental results to the simulations which is provided in Fig. 10 shows that these trends are reasonably captured. The URANS approach does not yield a finite probability, however, it succeeds in identifying the boundary conditions and locations which are most favorable for combustor light-off. For the case of the $40 \%$ ignition probability ( $4 \mathrm{~cm}$ axial position and $15 \mathrm{SLM}$ ), the temperature decline of the ignition kernel in the simulation is slower than at the failed cases at $1 \mathrm{~cm}$ axial position. This indicates that the conditions at $4 \mathrm{~cm}$ are not as detrimental as for the other failed cases at $1 \mathrm{~cm}$. It appears that this location represents a transition zone where ignition is still possible and might depend on stochastic velocity fluctuations which allow the flame kernel to either grow or quench. This information is lost due to the averaging of the turbulent velocity fluctuations in a URANS simulation.

\section{Conclusion}

We have demonstrated by the application of the proposed combustion model to several test cases, that the TFC model with the implementation of explicit species transport equations offers large benefits. In the course of this study we have provided details about how the species transport equations are linked to the reaction progress 
variable which define the physical properties, e.g. the temperature, in the extended model.

The first basic test case served as a validation of the preserved model ability to predict the correct flame stabilization and flame propagation characteristics. In the Bunsen burner configuration the Bunsen cone exhibited the correct angle for three different grid configurations, proving the model insensitivity to grid changes and elaborated the appropriate implementation of the model closure for the species and reaction progress variable. It was shown that issues in the standard model that arise due to the definition of the temperature upon the flame progress variable and the mixture fraction are resolved by coupling the temperature to the local chemical state.

In the further course of this study, it was established that the model is able to capture fuel or oxidizer sources within the flame. These can evolve through the evaporation of droplets in the flame or through diffusion through the flame front. This extends the models capabilities to multiphase flow conditions and avoids issues within mixing zones. Under multiphase flow conditions it is crucial to account for fuel sources. Fuel vapor if generated within the flame can have a large effect on the flame structure and lead to quenching as too rich conditions may develop, or to an enhancement of the flame in lean conditions. By means of a simple 1-D test case it was demonstrated that the model is also able to reproduce flame speed trends. These were recorded in a detailed chemistry simulation of laminar flames under multiphase flow conditions with respect to the overall equivalence ratio and the droplet diameter. The results show that the model can give an appropriate prediction of the flame growth prediction under these conditions.

In addition we have proposed an interface to trigger the flame with a secondary source term in the reaction progress variable equation which allows the flame to autoignite. This represents a significant advantage, as parameters such as the spark energy and the spark size can be included in the ignition study. The results of the spray combustor test cases have pointed out that this approach can be utilized to investigate the flame propagation following a successful ignition. It also offers a means to identify favorable boundary conditions and igniter locations needed for the combustor light-off.

Thus, the proposed extension of the TFC model provides an efficient numerical tool designed for parameters studies in the context of turbulent multiphase flow ignition.

\section{Acknowledgments}

The authors thank Rolls-Royce Deutschland for their funding. This work received also funding from the European Community through the project TIMECOP-AE (Project \# AST5-CT-2006-030828). It reflects only the author's views and the Community is not liable for any use that may be made of the information contained therein.

\section{References}

[1] M. Di Domenico, P. Gerlinger, M. Aigner, Development and validation of a new soot formation model for gas turbine combustor simulations, Combust. Flame 157 (2010) 246-258.

[2] C. K. Westbrook, F. L. . Dryer, Simplified reaction mechanisms for the oxidation of hydrocarbon fuels in flames., Combust. Sci. Tech. 27 (1981) 31-43.

[3] M. Germano, U. Piomelli, P. Moin, W. H. Cabot, A dynamic subgrid scale eddy viscosity model, Phys. Fluids 3 (1991) 17601765.

[4] P. Moin, K. Squires, W. Cabot, S. Lee, A dynamic subgrid-scale model for compressible turbulence and scalar transport, Phys. Fluids 3 (1991) 2746-2757.

[5] W. P. Jones, V. N. Prasad, LES-PDF simulation of a spark ignited turbulent methane jet, Proc. Combust. Inst. 33 (2011) 1355-1363.

[6] W. W. Kim, S. Menon, H. C. Mongia, Large eddy simulation of a gas turbine combustor, Combust. Sci. Tech. 43 (1999) 25-62.

[7] E. Riber, V. Moureau, M. Garcia, T. Poinsot, O. Simonin, Evaluation of numerical strategies for large eddy simulation of particulate two-phase recirculating flows, Journal of Comp. Phys. 228 (2009) 539-564.

[8] D. B. Spalding, Mixing and chemical reaction in steady confined turbulent flames, Thirteenth Symposium (International) on Combustion 13 (1971) 649-657.

[9] F. A. Williams, Turbulent Mixing in Nonreactive and Reactive Flows, Plenum Press, 1975.

[10] D. J. Fyffe, J. Moran, K. Kumaran, A. Al-Sharshani, R. Sadr, Effect of GTL-like jet fuel composition on GT engine altitude ignition performance. Part I: Combustor operability, Proceedings of the ASME Turbo Expo GT2011-45487.

[11] A. H. Lefebvre, Gas Turbine Combustion, Hemisphere Publishing Corporation, Washington, USA, 1983.

[12] P. A. Fokaides, N. Zarzalis, Lean-blowout dynamics of a liftedstabilized, non-premixed swirl flame, Third European Combustion Meeting.

[13] S. F. Ahmed, I. A. Bahane Ledezma, E. Mastorakos, Spark ignition in a turbulent shearless fuel-air mixing layer: Average flame growth rates, Proceedings of the 47th AIAA Aerospace Sciences Meeting, 2009, Orlando, FL (USA).

[14] S. F. Ahmed, R. Balachandran, T. Machione, E. Mastorakos, Spark ignition of turbulent nonpremixed bluff-body flames, Combust. Flame 151 (2007) 366-385.

[15] S. F. Ahmed, R. Balachandran, E. Mastorakos, Measurements of ignition probability in turbulent non-premixed counterflow flames, Proc. Combust. Inst. 31 (2006) 1507-1513.

[16] S. F. Ahmed, E. Mastorakos, Spark ignition of lifted turbulent jet flames, Combust. Flame 146 (2006) 215-231.

[17] T. Marchione, S. F. Ahmed, E. Mastorakos, Ignition of turbulent swirling n-heptane spray flames using single and multiple sparks, Combust. Flame 156 (2009) 166-180.

[18] S. R. Stow, M. Zedda, A. Triantafyllidis, A. Garmory, E. Mastorakos, T. Mosbach, Conditional moment closure LES modelling of an aero-engine combustor at relight conditions, Proceedings of the ASME Turbo Expo GT2011-46318.

[19] T. Mosbach, G. C. Gebel, P. Le Clercq, R. Sadr, K. Kannaiyan, A. Al-Sharshani, Investigation of GTL-like jet fuel composition on GT engine altitude ignition and combustion performance. Part II: Detailed diagnostics, Proceedings of the ASME Turbo Expo GT2011-45510.

[20] A. Neophytou, E. Richardson, E. Mastorakos, Spark ignition of turbulent recirculating non-premixed gas and spray flames: A model for predicting ignition probability, Combust. Flame 159 (2012) 1503-1522.

[21] J. Weckering, A. Sadiki, J. Janicka, E. Mastorakos, R. L. G. M. Eggels, A forced ignition probability analysis method using les and lagrangian particle monitoring, Proc. Combust. Inst. 33 (2011) 2919-2925.

[22] G. Lacaze, E. Richardson, T. Poinsot, Large eddy simulation of spark ignition in a turbulent methane jet, Combust. Flame 156 (2009) 1993-2009. 
[23] J. M. Boyde, M. Van Hove, M. Di Domenico, M. Aigner, The numerical generation of an ignition map by means of a turbulent flame speed closure approach for the configuration of a jet flame, Proceedings of the 50th ISABE conference, Gothenborg, Sweden ISABE-2011-1135.

[24] V. L. Zimont, Theory of turbulent combustion of homogeneous fuel mixtures at high Reynolds numbers, Comubstion, Explosion and Shockwaves 15/3 (1979) 305-311.

[25] V. L. Zimont, W. Polifke, M. Bettelini, W. Weisenstein, An efficient computational model for premixed turbulent combustion at high Reynolds numbers based on a turbulent flame speed closure, J. Eng. Gas. Turb. Power 120 (1998) 526-533.

[26] W. Polifke, P. Flohr, M. Brandt, Modeling of inhomogeneously premixed combustion with an extended TFC model, J. Eng. Gas. Turb. Power 124 (2002) 58-65.

[27] P. Flohr, H. Pitsch, A turbulent flame speed closure model for LES of industrial burner flows, Center of Turbulent Research. Proceedings of the Summer Program. (2000) 169-179.

[28] E. Knudsen, H. Pitsch, A dynamic model for the turbulent burning velocity for large eddy simulation for premixed combustion LES, Combust. Flame 154 (2008) 240-260.

[29] V. L. Zimont, A. N. Lipatnikov, A numerical model of premixed turbulent combustion and its validation, Chem. Phys. Reports 14(7) (1995) 993-1025.

[30] D. Cokljat, W. Polifke, P. Wild, A non-adiabatic method for calculation of premixed flames using a turbulent flame speed closure, 7th Int. Conference on Numerical Combustion, 1998, York, UK.

[31] J. P. Wood, J. B. Moss, Modelling partially premixed combustion using a turbulent burning velocity based closure, Proceedings of the European Combustion Meeting, 2003, Orléans, France.

[32] O. Colin, A. Benkenida, The 3-zones extended coherent flame model (ecfm3z) for computing premixed/diffusion combustion, Oil \& Gas Science and Technology - Rev. IFP 59/6 (2004) 593609.

[33] L. Vervisch, R. Hauguel, P. Domingo, R. M., Three facets of turbulent combustion modelling: DNS of premixed V-flame, LES of lifted nonpremixed flame and RANS of jet-flame, J. Turbulence 5 (2007) N4.

[34] J. A. Van Oijen, F. A. Lammers, L. P. H. De Goey, Modeling of complex premixed burner systems by using flamelet-generated manifolds, Combust. Flame 127 (2001) 2124-2134.

[35] J. H. Ferziger, M. Peric, Numerische Strömungsmechanik, Springer-Verlag, New York, 2008.

[36] S. B. Pope, Turbulent Flows, Cambridge University Press, UK, 2000.

[37] Y. Saad, Iterative methods for sparse linear systems, Society for Industrial and Applied Mathematics, Philadelphia, USA, 2003.

[38] J. M. Boyde, M. Di Domenico, B. E. Noll, M. Aigner, Spark ignition simulations and the generation of ignition maps by means of a turbulent flame speed closure approach, Proceedings of the ASME Turbo Expo GT2010-22211.

[39] J. M. Boyde, P. Le Clercq, M. Di Domenico, T. Mosbach, G. Gebel, M. Rachner, M. Aigner, Ignition and flame propagation along planar monodisperse droplet streams, Proceedings of the 49th AIAA Aerospace Sciences Meeting, 2011, Orlando, FL (USA).

[40] J. M. Boyde, P. Le Clercq, M. Di Domenico, M. Rachner, G. C. Gebel, T. Mosbach, M. Aigner, Validation of an ignition and flame propagation model for multiphase flows, Proceedings of the ASME Turbo Expo GT2011-45104.

[41] J. Boussinesq, Essai sur la théorie des eaux courantes, Memoires presentes par divers savants á l'Académie des Sciences XXIII 1 (1877) 1-680.

[42] K. N. C. Bray, P. A. Libby, G. Masuya, J. B. Moss, Turbulence production in premixed turbulent flames, Combust. Sci. Tech. 25 (1981) 127-140

[43] P. Bailly, M. Champion, D. Garreton, Counter-gradient diffusion in a confined turbulent premixed flame, Phys. Fluids 9 (1997) 766-774.
[44] S. B. Pope, Turbulent premixed combustion, Ann. Rev. Fluid Mech. 19 (1987) 237-270.

[45] D. Veynante, A. Trouve, K. N. C. Bray, T. Mantel, Gradient and counter-gradient scalar transport in turbulent premixed flames, J. Fluid Mech. 332 (1997) 263-293.

[46] L. Durand, Development, implementation and validation of LES models for inhomogeneously premixed turbulent combustion, Ph.D. thesis, University of Munich (2007).

[47] V. L. Zimont, Gas premixed combustion at high turbulence. Turbulent flame closure combustion model, Experimental Thermal and Fluid Science 21 (2000) 179-186.

[48] H. Eickhoff, Analysis of the turbulent burning velocity, Combust. Flame 128 (2002) 347-350.

[49] H. Schmid, P. Habisreuther, W. Leuckel, A model for calculating heat release in premixed turbulent flames, Combust. Flame 113 (1998) 79-91

50] N. Peters, The turbulent burning velocity for large-scale and small-scale turbulence, J. Fluid Mech. 384 (1999) 107-132.

51] F. Zhang, P. Habisreuther, M. Hettel, H. Bockhorn, Modelling of a premixed swirl-stabilized flame using a turbulent flame speed closure model in LES, Second ECCOMAS Conference on Computational Combustion.

[52] P. Le Clercq, N. Doué, M. Rachner, M. Aigner, Validation of a multicomponent-fuel model for spray computation, Proceedings of the 47th AIAA Aerospace Sciences Meeting, 2009, Orlando, FL (USA).

[53] Z. Tan, R. D. Reitz, An ignition and combustion model based on the level-set method for spark ignition engine multidimensional modeling, Combust. Flame 145 (2006) 1-15.

[54] M. Ihme, H. Pitsch, Prediction of extinction and reignition in nonpremixed turbulent flames using a flamelet/progress variable model 1. A priori study and presumed PDF closure, Combust. Flame 155 (2008) 70-89.

[55] J.-B. Michel, O. Colin, C. Angelberger, D. Veynante, Using the tabulated diffusion flamelet model ADF-PCM to simulate a lifted methane-air jet flame, Combust. Flame 156 (2009) 13181331.

[56] B. J. McBridge, M. J. Zehe, S. Gordon, NASA Glenn coefficients for calculating thermodynamic properties of individual species, Tech. rep., TP-2002-21155. NASA (2002).

[57] J. M. Boyde, A. Fiolitakis, M. Di Domenico, M. Aigner, Correlations for the laminar flame speed, adiabatic flame temperature and ignition delay time for methane, ethanol and n-decane, Proceedings of the 49th AIAA Aerospace Sciences Meeting, 2011, Orlando, FL (USA).

[58] D. Bradley, F. K.-K. Lung, Spark ignition and the early stages of turbulent flame propagation, Combust. Flame 69 (1987) 7193.

59] B. Lewis, von Elbe G., Combustion, Flames and Explosion of Gases, Academic Press, New York and London, 1961.

[60] R. Maly, Ignition model for spark discharges and the early phase of flame front growth, Proc. Combust. Inst. 18 (1981) 17471754 .

[61] D. Bradley, C. G. W. Sheppard, I. M. Suardjaja, R. Woolley, Fundamentals of high-energy spark ignition with lasers, Combust. Flame 156 (2009) 55-77.

[62] N. Chakraborty, E. Mastorakos, R. S. Cant, Effects of turbulence on spark ignition in inhomogeneous mixtures: a direct numerical simulation (DNS) study, Combust. Sci. Tech. 179 (2007) 293-317.

[63] G. Lacaze, B. Cuenot, T. Poinsot, M. Oschwald, Large eddy simulation of laser ignition and compressible reacting flow in a rocket-like configuration, Combust. Flame 156 (2009) 11661180

[64] T. Kravchik, E. Sher, Numerical modeling of spark ignition and flame initiation in a quiescent methane-air mixture, Combust. Flame 99 (1994) 635-643.

[65] S. Pischinger, J. B. Heywood, A model for flame kernel development in a sprak-ignition engine, Proc. Combust. Inst. 22 (1990) 1033-1040.

[66] G. C. Gebel, T. Mosbach, W. Meier, M. Aigner, Laser-induced 
ignition of kerosene in a model combustor, Proceedings of the European Combustion Meeting, 2011, Cardiff, Ireland.

[67] G. C. Gebel, T. Mosbach, W. Meier, M. Aigner, S. Le Brun, An experimental investigation of kerosene droplet breakup by laserinduced blast waves, Proceedings of the ASME Turbo Expo GT2012-68963.

[68] T. Mosbach, G. C. Gebel, Report on the experiments at the labscale combustor (D2.2.3b), Tech. rep., TIMECOP-AE Project no: AST5-CT-2006-030828 (2010).

[69] E. Bluemcke, Turbulente partikeldispersion in eingeschlossenen drallstroemungen, Ph.D. thesis, DLR, German Aerospace Center, Institute of Propulsion Technology (1992).

[70] W. A. Sirignano, Fluid Dynamics and Transport of Droplets and Sprays, Cambridge University Press, 1999.

[71] B. Abramzon, W. A. Sirignano, Droplet vaporization model for spray combustion calculations, Int. J. Heat Mass Transfer 32 (1989) 1605-1618.

[72] R. W. Bilger, A mixture fraction framework for the theory and modeling of droplets and sprays, Combust. Flame 158 (2011) 191-202.

[73] M. Mortensen, R. W. Bilger, Derivation of the conditional moment closure equations for spray combustion, Combust. Flame 156 (2009) 62-72.

[74] M. Liberman, Introduction to Physics and Chemistry of Combustion: Explosion, Flame, Detonation, Springer-Verlag, Berlin, 2008.

[75] N. Peters, Technische Verbrennung, University of Aachen, Course Material, 2006.

[76] G. P. Smith, M. Golden, D. M. Frenklach, N. W. Moriarty, B. Eiteneer, M. Goldenberg, C. T. Bowman, R. K. Hanson, S. Sond, W. C. j. Gardiner, V. V. Lissianske, Z. Qin, GRI 3.0, http://www.me.berkely.edu/gri_mech/.

[77] U. C. Müller, M. Bollig, N. Peters, Approximations for burning velocities and Markstein numbers for lean hydrocarbon and methanol flames, Combust. Flame 108 (1997) 349-356.

[78] A. Neophytou, E. Mastorakos, Simulations of laminar flame propagation in droplet mists, Combust. Flame 156 (2009) 16271640 .

[79] T. Mosbach, GerMaTec - Experimental investigation and validation measurements, Tech. rep., DLR Stuttgart (2010).

[80] J. M. Boyde, Y. Schneider-Kühnle, M. Aigner, Lufo IV project GerMaTec, Tech. rep., DLR-VT (2011). 
Appendix A. Correlations for Laminar Flame Speed and Auto-Ignition Time

Table 10: Coefficients of the polynomial for the laminar flame speed of methane.

Coefficients for the Laminar Flame Speed

General Form: $S_{\mathrm{L}}=C_{1}+C_{2} \phi+C_{3} \phi^{2}+C_{4} \phi^{3}+C_{5} \phi^{4}$

Correlation for $C_{i}$

Formulation: $C_{i}=C_{i, 1}^{\star}+C_{i, 2}^{\star} T+C_{i, 3}^{\star} T^{2}+C_{i, 4}^{\star} T^{3}+C_{i, 5}^{\star} T^{4}+C_{i, 6}^{\star} p+C_{i, 7}^{\star} p \cdot T+C_{i, 8}^{\star} p \cdot T^{2}+C_{i, 9}^{\star} p^{2} \cdot T^{2}$

Note, T/300 [K], p/1e5 [Pa].

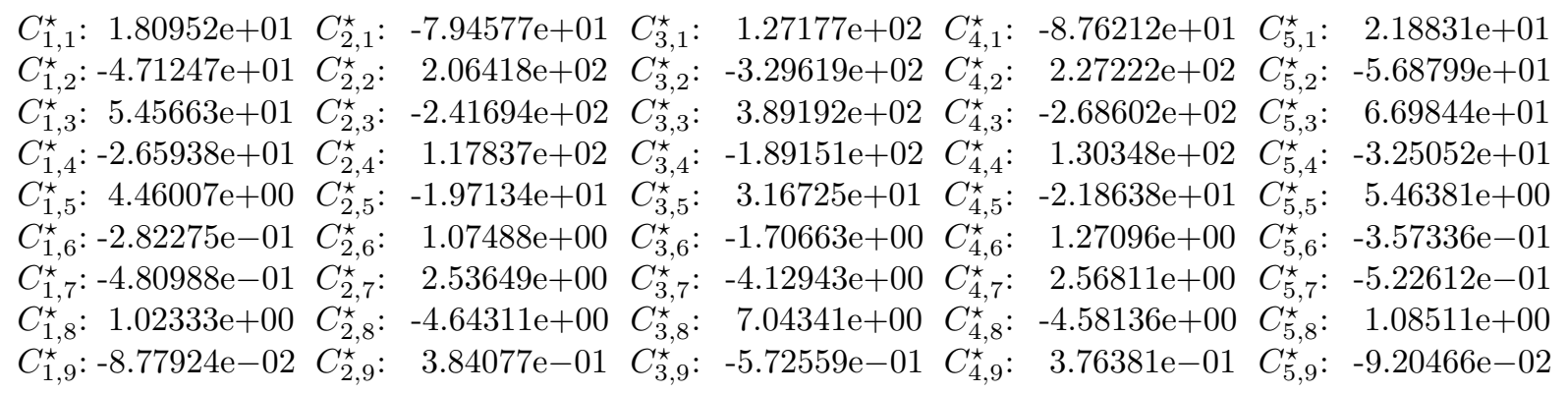

Table 11: Coefficients of the polynomial for the ignition delay time of methane.

\section{Coefficients for the Ignition Delay Time}

General Form: $t_{\mathrm{i}}=C_{1}+C_{2} \phi$

Correlation for $C_{i}$

Formulation: $C_{i}=C_{i, 1}^{\star}+C_{i, 2}^{\star} \cdot 1 / T+C_{i, 3}^{\star} \cdot 1 / T^{2}+C_{i, 4}^{\star} \cdot 1 / T^{3}+C_{i, 5}^{\star} \cdot 1 / T^{4}+C_{i, 6}^{\star} \cdot 1 / p$

$+C_{i, 7}^{\star} \cdot 1 /(p \cdot T)+C_{i, 8}^{\star} \cdot 1 /\left(p \cdot T^{2}\right)+C_{i, 9}^{\star} \cdot 1 /\left(p \cdot T^{3}\right)+C_{i, 10}^{\star} \cdot 1 /\left(p \cdot T^{4}\right)$

Note, T/1000 [K], p/1e5 [Pa].

Note, coefficients are written in row formation.

$C_{1,1}^{\star}: 2.39005 \mathrm{e}-02 \quad C_{1,2}^{\star}:-1.74903 \mathrm{e}-01 \quad C_{1,3}^{\star}: \quad 4.79014 \mathrm{e}-01 \quad C_{1,4}^{\star}: \quad-5.82448 \mathrm{e}-01 \quad C_{1,5}^{\star}: \quad 2.65583 \mathrm{e}-01$

$C_{1,6}^{\star}: 3.39938 \mathrm{e}-01 \quad C_{1,7}^{\star}: \quad-2.26768 \mathrm{e}+00 \quad C_{1,8}^{\star}: \quad 5.68183 \mathrm{e}+00 \quad C_{1,9}^{\star}: \quad-6.34326 \mathrm{e}+00 \quad C_{1,10}^{\star}: 2.66631 \mathrm{e}+00$

$C_{2,1}^{\star}: 1.75173 \mathrm{e}-02 \quad C_{2,2}^{\star}:-1.22666 \mathrm{e}-01 \quad C_{2,3}^{\star}: \quad 3.26076 \mathrm{e}-01 \quad C_{2,4}^{\star}:-3.90313 \mathrm{e}-01 \quad C_{2,5}^{\star}: \quad 1.77613 \mathrm{e}-01$

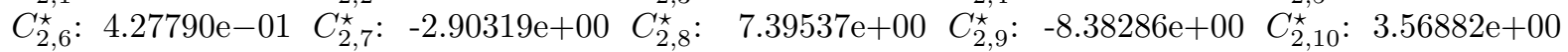




\section{Coefficients for the Laminar Flame Speed}

General Form: $S_{\mathrm{L}}=C_{1}+C_{2} \phi+C_{3} \phi^{2}+C_{4} \phi^{3}+C_{5} \phi^{4}$

Correlation for $C_{i}$

Formulation: $C_{i}=C_{i, 1}^{\star}+C_{i, 2}^{\star} T+C_{i, 3}^{\star} T^{2}+C_{i, 4}^{\star} T^{3}+C_{i, 5}^{\star} T^{4}+C_{i, 6}^{\star} p+C_{i, 7}^{\star} p \cdot T+C_{i, 8}^{\star} p \cdot T^{2}+C_{i, 9}^{\star} p^{2} \cdot T^{2}$

Note, T/300 [K], p/1e5 [Pa].

\begin{tabular}{|c|c|c|c|c|c|c|c|}
\hline$C_{1,1}^{\star}:-6.09241 \mathrm{e}+01$ & $C_{2,1}^{\star}:$ & & $C_{3,1}^{\star}:$ & & $C_{4,1}^{\star}:$ & $C_{5,1}^{\star}:$ & \\
\hline$C_{1,2}^{\star}: 1.86125 \mathrm{e}+02$ & $C_{2,2}^{\star}:$ & $-7.96783 \mathrm{e}+02$ & $C_{3,2}^{\star}:$ & $1.23133 \mathrm{e}+03$ & $C_{4,2}^{\star}:$ & $-8.12882 \mathrm{e}+02 C_{5,2}^{\star}:$ & \\
\hline$C_{1,3}^{\star}:-1.94090 \mathrm{e}+02$ & $C_{2,3}^{\star}:$ & $8.30943 \mathrm{e}+02$ & $C_{3,3}^{\star}:$ & $-1.28441 \mathrm{e}+03$ & $C_{4,3}^{\star}:$ & $8.49108 \mathrm{e}+02 C_{5,3}^{\star}:$ & $\mathrm{e}+02$ \\
\hline$C_{1,4}^{\star}: 9.00070 \mathrm{e}+01$ & $C_{2,4}^{\star}:$ & $-3.86284 \mathrm{e}+02$ & $C_{3,4}^{\star}:$ & $5.98929 \mathrm{e}+02$ & $C_{4,4}^{\star}:$ & $-3.96813 \mathrm{e}+02 C_{5,4}^{\star}:$ & $\vdash 01$ \\
\hline$C_{1,5}^{\star}:-1.56202 \mathrm{e}+01$ & $C_{2,5}^{\star}:$ & 6.7 & $C_{3,5}^{\star}:$ & -1.0 & $C_{4,5}^{\star}:$ & $01 C_{5,5}^{\star, 4}:$ & \\
\hline $5767 e+00$ & $C_{2,6}^{\star}:$ & $-5.62723 \mathrm{e}+00$ & & 8.51 & & $+00 C_{5,6}^{\star}:$ & +00 \\
\hline$C_{17}^{\star}:-2.07916 \mathrm{e}+00$ & $C_{2,7}^{\star}:$ & $8.79306 \mathrm{e}+00$ & $C_{37}^{\star \star 0}:$ & $-1.34222 \mathrm{e}+01$ & $C_{4,7}^{\star}:$ & $666 \mathrm{e}+00 \quad C_{57}^{\star}:$ & +00 \\
\hline$C_{1,8}^{\star}: 1.89322 \mathrm{e}+00$ & $C_{2,8}^{\star}:$ & $-7.95661 \mathrm{e}+00$ & $C_{3,8}^{\star}:$ & & $C_{4,8}^{\star}:$ & $+00 C_{5,8}^{\star}:$ & +00 \\
\hline$C_{1,9}^{\star}:-1.99070 \mathrm{e}-01$ & $C_{2,9}^{\star}:$ & $8.24998 \mathrm{e}-01$ & $C_{3,9}^{\star}:$ & $-1.18174 \mathrm{e}+00$ & $C_{4,9}^{\star}:$ & $7.16808 \mathrm{e}-01 \quad C_{5,9}^{\star}:$ & $-1.57116 \mathrm{e}-01$ \\
\hline
\end{tabular}

Table 13: Coefficients of the polynomial for the ignition delay time of n-decane.

\section{Coefficients for the Ignition Delay Time}

General Form: $t_{\mathrm{i}}=C_{1}+C_{2} \phi$

Correlation for $C_{i}$

Formulation: $C_{i}=C_{i, 1}^{\star}+C_{i, 2}^{\star} \cdot 1 / T+C_{i, 3}^{\star} \cdot 1 / T^{2}+C_{i, 4}^{\star} \cdot 1 / T^{3}+C_{i, 5}^{\star} \cdot 1 / T^{4}+C_{i, 6}^{\star} \cdot 1 / p$ $+C_{i, 7}^{\star} \cdot 1 /(p \cdot T)+C_{i, 8}^{\star} \cdot 1 /\left(p \cdot T^{2}\right)+C_{i, 9}^{\star} \cdot 1 /\left(p \cdot T^{3}\right)+C_{i, 10}^{\star} \cdot 1 /\left(p \cdot T^{4}\right)$

Note, T/1000 $[\mathrm{K}], \mathrm{p} / 1 \mathrm{e} 5[\mathrm{~Pa}]$.

Note, coefficients are written in row formation.

$C_{1,1}^{\star}: 5.41490 \mathrm{e}-02 \quad C_{1,2}^{\star}: \quad-3.44915 \mathrm{e}-01 \quad C_{1,3}^{\star}: \quad 8.21339 \mathrm{e}-01 \quad C_{1,4}^{\star}: \quad-8.67036 \mathrm{e}-01 \quad C_{1,5}^{\star}: \quad 3.42581 \mathrm{e}-01$ $C_{1,6}^{\star}:-4.84663 \mathrm{e}-02 \quad C_{1,7}^{\star}: \quad 2.82626 \mathrm{e}-01 \quad C_{1,8}^{\star}: \quad-6.05832 \mathrm{e}-01 \quad C_{1,9}^{\star}: \quad 5.61393 \mathrm{e}-01 \quad C_{1,10}^{\star}:-1.87151 \mathrm{e}-01$ $C_{2,1}^{\star}:-6.57936 \mathrm{e}-02 \quad C_{2,2}^{\star}: \quad 4.16056 \mathrm{e}-01 \quad C_{2,3}^{\star}: \quad-9.83080 \mathrm{e}-01 \quad C_{2,4}^{\star}: \quad 1.02895 \mathrm{e}+00 \quad C_{2,5}^{\star}:-4.02643 \mathrm{e}-01$ $C_{2,6}^{\star}: \quad 7.31715 \mathrm{e}-02 \quad C_{2,7}^{\star}: \quad-4.47257 \mathrm{e}-01 \quad C_{2,8}^{\star}: \quad 1.01743 \mathrm{e}+00 \quad C_{2,9}^{\star}: \quad-1.01960 \mathrm{e}+00 \quad C_{2,10}^{\star}: \quad 3.79219 \mathrm{e}-01$ 